16 Casey Giordano, Deniz S. Ones, and Niels G. Waller, Department of Psychology, University

17 of Minnesota; Kevin C. Stanek, preValio, LLC.

18 Correspondence concerning this article should be addressed to Casey Giordano or Deniz S.

19 Ones, Department of Psychology, University of Minnesota, 75 East River Road, Minneapolis,

20 MN 55455. E-mails: giord023@umn.edu or deniz.s.ones-1@tc.umn.edu.

21 This research was partially supported by the Auke Tellegen Fellowship in Applied

22 Personality Assessment awarded to Casey Giordano. 
Abstract

24 We provide an overview of and guidance for applying exploratory bifactor models to vocational

25 research. First, we describe bifactor models and highlight their potential and actual applications

26 in vocational psychology. Second, we review the theoretical bases of bifactor models and offer

27 methodological guidance to correctly implement and interpret these models in practice. Third,

28 we estimate a bifactor model in two vocational datasets to illustrate the concepts reviewed in this

29 manuscript. The resulting models highlight novel insights in careers research (e.g.,

30 developmental performance feedback and personality [conscientiousness] modeling) that are

31 made possible by leveraging bifactor measurement models. Overall, this manuscript provides a

32 useful introduction to bifactor models to facilitate vocational behavior scholars and practitioners

33 in thoughtfully producing and consuming bifactor models in their own research. 


\section{Exploratory Bifactor Measurement Models in Vocational Behavior Research}

The field of psychology is in the midst of a bifactor model renaissance. Evincing this

renaissance, Reise's (2012) rediscovery of bifactor models has quickly become a citation classic,

37 amassing over 700 citations $^{1}$ in a few years - eclipsing that of Schmid and Leiman's (1957)

38 seminal article more than half a century after its publication. This precipitous resurgence of

39 bifactor models has spurred rapid methodological advancements — particularly in the domain of

40 exploratory bifactor analysis (Giordano \& Waller, 2020). Exploratory bifactor analyses can

41 contribute to and support the refinement of multidimensional psychological theories dominating

42 vocational behavior research of the past half century.

43 The purpose of this manuscript is to introduce exploratory bifactor analysis to the broader

44 audience of vocational behavior researchers and to provide them with the necessary tools to

45 apply bifactor models to their own work. To achieve these goals, we first provide a conceptual

46 overview of bifactor models. Next, we illustrate the utility and broad applicability of exploratory

47 bifactor models with an emphasis on career and vocational behavior research domains. Then,

48 using two datasets, we demonstrate how a researcher might leverage bifactor models to answer

49 important substantive questions. To facilitate the proper application of the ideas discussed in this

50 manuscript, the online supplemental materials include the statistical code and data used to

51 estimate exploratory bifactor models and interpret the results. Through this manuscript, we hope

52 to aid vocational behavior researchers to correctly (a) understand the strengths and limitations of

${ }^{1}$ Reise (2012) has been cited 711 times according to Web of Science, accessed on February $18^{\text {th }}, 2020$. 
53 exploratory bifactor estimation methods, (b) estimate exploratory bifactor models in their own

54 research, and (c) interpret results from a bifactor measurement model.

Background, Applicability, and Applications of Bifactor Measurement Models

\section{Background}

Exploratory bifactor analysis refers to a class of models within the broader exploratory

58 factor analytic domain (for historical reviews, see Carroll, 1993, Ch. 2; Giordano \& Waller,

59 2020). At its core, factor analysis is a data-driven approach to modeling latent factors as

60 determinants of observed data (e.g., responses to career satisfaction items). To use a linear

61 regression analogy, latent factors are the independent variables that predict variation in the

62 dependent variables (i.e., observed variables). Holzinger's (1935, 1936, 1937, 1945; Holzinger \&

63 Swineford, 1937) bifactor model was designed to account for variance in observed variables

64 from the effects of three types of latent factors: (a) a general factor that influences all observed

65 variables, (b) multiple group factors ${ }^{2}$ that influence a subset of the observed variables, and (c) a

66 set of uniqueness factors, each one of which captures variance unique to an observed variable.

67 To visualize an example bifactor measurement model, see the path diagram in Figure 1.

68 Conceptually, the bifactor model represents a marriage between Spearman's (1904) general

69 factor model and Thurstone's $(1934,1947)$ multiple factors model. In most applications of the

70 bifactor model, all latent factors are mutually orthogonal (uncorrelated; for an exception, see

71 Jennrich \& Bentler, 2012).

\footnotetext{
${ }^{2}$ Many bifactor applications call these factors “specific factors.” In this paper, we defer to Holzinger's (1937) nomenclature of group factors because, in factor analysis, "specific factors" are a component of uniqueness factors.
} 
Insert Figure 1 here

As a class of models within exploratory factor analysis, it is helpful to distinguish

76 between two types of bifactor models. Namely, constrained, hierarchical bifactor models and

77 unconstrained, non-hierarchical bifactor models. These model types differ in the dimensionality

78 of (a) the bifactor loadings matrix and (b) the estimated factor scores. Although hierarchical and

79 non-hierarchical bifactor solutions can produce similar patterns of factor loadings (Giordano \&

80 Waller, 2020), the aforementioned dimensionality differences have important ramifications for

81 both theoretical interpretations and practical applications (we address these topics more fully in

82 later sections).

\section{Hierarchical and Non-Hierarchical Bifactor Models}

The main difference between hierarchical and non-hierarchical bifactor models is the

85 presence or absence of additional model constraints (Yung, Thissen, \& McLeod, 1999; see also,

86 Gignac, 2016). To understand the implications of these constraints, it is helpful to understand

87 how a constrained, hierarchical bifactor model is estimated. Traditionally, hierarchical bifactor

88 models have been conceptualized as re-expressions of higher-order, common factor models (e.g.,

89 Schmid \& Leiman, 1957; Thomson, 1951; Thurstone, 1947). Higher-order factor analyses seek

90 to explain first-order factor correlations (i.e., from an obliquely-rotated, correlated-factors

91 model) by one or more higher-order factors. When one higher-order factor sufficiently accounts

92 for the lower-order factor correlations, the higher-order factor is often called a general factor.

93 Structurally, the general factor (or multiple general factors) putatively influences the lower-order

94 latent factors, which in turn influence the observed variables. Stated differently, the general 
95 factor has an indirect (i.e., mediated) effect on each observed variable. When re-expressing a

96 higher-order model as a hierarchical bifactor model, these indirect effects result in a constrained

97 bifactor loadings matrix that is rank-deficient (Waller, 2018). In common parlance, this means

98 that the general factor loadings are an exact linear combination of the group factor loadings. In

99 contrast to hierarchical models, the general factor in non-hierarchical bifactor models has a direct

100 (i.e., non-mediated) effect on each observed variable. Thus, non-hierarchical bifactor models

101 produce unconstrained bifactor loadings matrices that are full-rank in the sense that the general

102 factor loadings are (statistically and theoretically) independent from the group factor loadings.

103 By including hierarchical and non-hierarchical solutions under the bifactor moniker, this

104 paper diverges from some researchers that only consider non-hierarchical bifactor solutions in

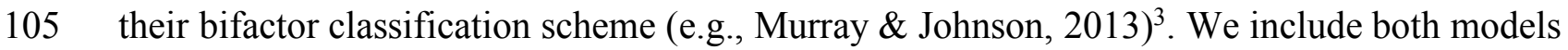

106 under our bifactor umbrella for two reasons. First, we previously described bifactor models as

107 those including (a) a general factor (or multiple general factors), (b) multiple group factors, and

108 (c) uniqueness factors. No stipulations were made about constraints on the estimated bifactor

109 loadings structure. Second, empirically ascertaining whether one's observed data adhere to a

110 hierarchical or a non-hierarchical bifactor model can be exceedingly difficult (e.g., Giordano \&

111 Waller, 2020; Greene et al., 2019; Mulaik \& Quartetti, 1997; Rindskopf \& Rose, 1988, Yang,

112 Spirtes, Scheines, Reise, \& Mansolf, 2017). Related to this latter point, previous research has

113 quantitatively examined the similarity of hierarchical and non-hierarchical solutions. For

\footnotetext{
${ }^{3}$ Several researchers use the term 'hierarchical bifactor model' to refer to what we call an unconstrained nonhierarchical bifactor model (e.g., Gignac, 2008, 2016). Whereas in this paper we use the term 'hierarchical' to reference a multi-order factor solution (i.e., a hierarchy of factors), the other use of 'hierarchical' refers to a breadth factor (i.e., a factor that influences many variables).
} 
114 example, in a simulation comparing 162 non-hierarchical bifactor models to their closest

115 hierarchical analog, Giordano and Waller (2020) found a median congruence coefficient ${ }^{4}$ of

$116.995(\min =.97)$. In other words, on average, the non-hierarchical bifactor pattern and its

117 hierarchical counterpart were virtually indistinguishable in these simulations.

118 Whereas the empirical differences between (constrained) hierarchical and (unconstrained)

119 non-hierarchical bifactor models may be small, the theoretical differences can be substantial.

120 Namely, the relationship between latent factors and observed variables differs across the two

121 models. In the constrained hierarchical model, the general factor's influence on the observed

122 variables is mediated through the first-order factors. Alternatively, in the unconstrained non-

123 hierarchical model, the general factor directly influences the observed variables. In substantive

124 terms, these models differ in the causal pathways (representing mediated and direct effects)

125 between factors and observed variables. Moreover, the two models also differ in how the group

126 factors are defined. Specifically, group factors in the hierarchical model are residualized, lower-

127 order factors that are created by partialling out the effects of the general factor from the original,

128 correlated lower-order factors. In contrast to that approach, the group factors in the non-

129 hierarchical model are directly defined to be orthogonal to the general factor (cf. Abad et al.,

130 2017; Giordano \& Waller, 2020).

131 Taken together, due to the combination of (a) minor empirical differences and (b)

132 meaningful theoretical differences between the two models, we believe that deciding whether to

133 estimate a hierarchical or a non-hierarchical bifactor model should not be based on fit indices

\footnotetext{
${ }^{4}$ A congruence coefficient (Lorenzo-Seva \& ten Berge, 2006) is a common index for measuring the similarity between two factor solutions. Congruence coefficient values (that range from 0 to 1 ) over .95 are suggestive that the two solutions are functionally equivalent (Lorenzo-Seva \& ten Berge, 2006).
} 
134 alone. Rather, we recommend that researchers draw upon theoretical frameworks and domain

135 knowledge when deciding between the two types of bifactor models (see also, Preacher, Zhang,

136 Kim, \& Mels, 2013). Exemplifying this approach, ${ }^{5}$ Beaujean (2015) reviewed Carroll's (e.g.,

137 1993) highly influential work on the structure of cognitive abilities and argued that the cognitive

138 abilities domain is best represented by a non-hierarchical bifactor model (cf. McGrew, 2005 for

139 an opposing view). Other researchers (e.g., Digman, 1997; Stanek \& Ones, 2018) have suggested

140 that many Big Five personality traits are consistent with higher-order factor models and, thus,

141 could be profitably modelled by hierarchical bifactor models.

\section{Fitting Exploratory Bifactor Models to Vocational Data}

143 One reason for the renewed interest in both constrained and unconstrained bifactor

144 measurement models is that both models produce orthogonal factors that are (often) easily

145 interpretable. Among these orthogonal factors, in most substantive domains, the general factor

146 often accounts for the lion's-share of the covariation among the observed variables (e.g., items,

147 item clusters, scales, etc.). After partialling out the effects of the general factor, the residual

148 correlations are presumably due to the group factors (Holzinger \& Swineford, 1937). In

149 vocational research, group factors could represent substantive constructs (e.g., facets of a

150 construct), methodological factors (e.g., positive and negative valence from positively and

151 negatively worded items), contextual domain effects (e.g., the same construct manifesting in

152 work, educational, personal life), or temporal influences (e.g., developmental stages of a

153 construct; aging effects) on the observed variables. To illustrate these ideas, Figure 1 shows how

\footnotetext{
${ }^{5}$ We provide these examples of hierarchical versus non-hierarchical representations purely for illustrative purposes. It is beyond the scope of this manuscript to make definitive claims about the appropriateness of specific bifactor models in substantive domains.
} 
154 the bifactor model can be applied to account for the many potential sources of variance in 155 vocational data.

156 To date, bifactor models have been overwhelmingly applied to datasets wherein group

157 factors represent systematic construct variation beyond that of the general factor. This has been 158 particularly true in the domain of cognitive ability (e.g., Carroll, 1993; Cucina \& Byle, 2017).

159 Though such study designs serve an important role in advancing our understanding of various 160 construct domains, bifactor models can be fruitfully applied to a broader range of study designs.

161 For example, Levin (1973) applied a bifactor model to a multitrait-multimethod study design 162 where four leadership criteria were assessed by self, peer, and observer reports. Modeling the 163 rating sources as group factors allows one to specifically parse out (a) the shared variance across 164 rating sources (i.e., a general leadership factor) and (b) the unshared variance that is unique to 165 each source (e.g., source-specific perspectives in ratings). The resulting bifactor model clearly 166 showed that observer ratings are more strongly influenced by a general leadership factor. In 167 contrast, self-ratings were influenced more heavily by source-specific perspective effects with a 168 comparatively weaker influence from the general leadership factor.

169 Like the leadership domain, most, if not all, constructs in vocational research are 170 multidimensional. A common yet important question in multidimensional variable domains is the 171 relative strength of the general factor (e.g., the strength of a general satisfaction factor) versus the 172 strength of more narrowly defined group factors (e.g., satisfaction with advancement, pay, 173 meaningfulness, and work-life balance). Such questions are easily addressed via bifactor 174 measurement models that partition latent factor variance into uncorrelated general and group 175 factors. For example, a 2019 issue of the Journal of Vocational Behavior, examined this question 176 in three studies pertaining to the Psychology of Working Theory (Duffy, Blustein, Diemer, \& 
177 Autin, 2016). These studies investigated, in three separate countries, how work can impact the 178 fulfillment of one's basic human needs. In Italy, Portugal, and Brazil, the general 'decent work'

179 factor accounted for $59 \%, 52 \%$, and $65 \%$ of the total observed scale variance, respectively (Di

180 Fabio \& Kenny, 2019; Ferreira, et al., 2019; Ribeiro, Teixeira, \& Ambiel, 2019). The cumulative

181 effect of the 'decent work' subdimensions - that is, safe conditions, access to healthcare,

182 adequate compensation, free time and rest, and complementary values - accounted for 36\%,

$18341 \%$, and $30 \%$ of the variance for Italy, Portugal, and Brazil, respectively. Thus, whereas the

184 'decent work' subdimensions are all positively correlated (i.e., a strong general factor is present),

185 there is meaningful differentiation among the group factors. We note that none of the three

186 studies theoretically justified whether a constrained (hierarchical) or unconstrained (non-

187 hierarchical) bifactor model would fit better.

Another application of bifactor modeling in career and vocational psychology examined

189 the differentiation of occupational interests (Toker \& Ackerman, 2012). Specifically, Toker and

190 Ackerman were concerned with science, technology, engineering, and mathematics (STEM)

191 students and investigated how STEM students differ in their interest for complex careers. These

192 authors applied a bifactor model with a general 'complexity interest' factor as well as group

193 factors representing numerical complexity, symbolic complexity, spatial complexity, and idea

194 complexity. Further analysis of the original factor solution (see our online supplement) found

195 that the general 'complexity interest' factor accounted for $80 \%$ of the total observed score

196 variance whereas the remaining group factors collectively accounted for $18 \%$ of the total

197 observed variance. Simply put, in this example, $80 \%$ of the variance in an observed scale-score

198 was comprised of general factor variance. Some authors would claim that values in this range are

199 prima facie evidence for a unidimensional measure (Rodriguez, Reise, \& Haviland, 2016). 
200 Importantly, however, group factors have stronger effects in their associated subscale scores but

201 these effects shrink in total scale scores by nature of (a) adding items from unrelated group

202 factors and (b) a general factor that impacts all items.

203 The 'decent work' and 'occupational complexity interest' domains are only two

204 demonstrations of the utility for applying bifactor measurement models. Many prominent

205 variables and criteria of interest are also multidimensional: job performance (e.g., J. P. Campbell

206 \& Wiernik, 2015; Viswesvaran \& Ones, 2000), organizational citizenship (e.g., LePine, Erez, \&

207 Johnson, 2002), transformational leadership (e.g., Judge \& Piccolo, 2004), emotional labor (e.g.,

208 Morris \& Feldman, 1996), burnout (e.g., Demerouti, Bakker, Varkadou, \& Kantas, 2003), job

209 satisfaction (e.g., Locke, 1969), employability (e.g., Fugate, Kinicki, \& Ashforth, 2004), career

210 success (e.g., Arthur, Khapova, \& Wilderom, 2005), quality of life (Chen, West, \& Sousa, 2006),

211 and career adaptability (e.g., Zacher, 2014), among numerous others. Likewise, assessments of

212 key explanatory variables are often multidimensional and are thus well represented by bifactor

213 measurement models. Examples include many personality assessments (e.g., McCrae \& Costa,

214 2004; Stanek \& Ones, 2018), interest measures (e.g., D. P. Campbell \& Holland, 1972),

215 cognitive ability tests (e.g., Carroll, 1993), affect scales (e.g., Watson, Clark, \& Tellegen, 1988),

216 situational/contextual characteristics (e.g., Rauthmann, Gallardo-Pujol, Guillaume, et al., 2014),

217 inventories of occupational constraints and demands (e.g., Karasek, 1979), measures of

218 organizational support constructs (e.g., Rhoades \& Eisenberger, 2002), and many others. Each of

219 these domains are well suited for bifactor modeling.

220 The extant literature suggests that bifactor measurement models can aid in understanding

221 subdimensions of hierarchical construct domains. However, there are many other ways in which

222 to conceptualize and model the group factors in a bifactor model (see Figure 1). For example, 
223 group factors can represent method-specific effects in a multimethod study design (e.g., Levin,

224 1973; McAbee \& Connelly, 2016), an approach we take in Example 1 below. Other method

225 effects, such as positively and negatively worded items, can be modeled to partition variance into

226 a substantive general factor of the focal construct and group factors associated with the

227 potentially contaminating effects of item keying (e.g., "I enjoy my work environment" and "I

228 loathe my work tasks" are oppositely-keyed items of job satisfaction). Developmental effects

229 might also be modeled with bifactor models. Consider measures of vocational interests in

230 adolescence, adulthood, and older age. Longitudinal interest data from these developmental

231 stages can be modeled to identify, for example, a general social interest factor alongside life-

232 stage-limited social interests (i.e., group factors corresponding to each life stage). Life domain or

233 context effects can also constitute group factors (e.g., Stanek, Ones, \& McGue, 2017). To

234 provide another example in the interest domain, previous research has found that "vocational,

235 leisure, and family interests of adults are strongly intercorrelated" (Gaudron \& Vautier, 2007, p.

236 568), even after accounting for a common methods factor. When applied to, say, realistic

237 interests (e.g., D. P. Campbell \& Holland, 1972), a bifactor model could provide insights into the

238 amount of variation that is due to the global realistic interest factor as well as specific group

239 factors, such as realistic vocational, realistic leisure, and realistic family interests. Does the

240 general 'realistic interest' factor account for the most variance or do people meaningfully

241 differentiate their interests according to specific contexts? Here, a bifactor model can be

242 leveraged to advance developmental and individual difference theories of vocational interests.

243 These are just a few examples of novel bifactor applications to address unanswered substantive

244 questions in psychological domains. 


\section{Implementing Bifactor Measurement Models:}

\section{Methodological Decisions and Their Consequences}

\section{Estimating Exploratory Bifactor Models}

Like other multivariate analyses, a bifactor analysis requires numerous methodological

249 choices that can influence the quality of the obtained solution. Although some choices might not

250 meaningfully alter the obtained pattern of bifactor loadings, other choices during model

251 estimation can prominently impact obtained bifactor solutions. Here we highlight a few key

252 methodological decisions and issues relevant to bifactor modeling.

253 Deciding the Number of Latent Factors to Model. An influential, early decision in the

254 bifactor modeling process is deciding on the number of latent factors to model (Preacher, Zhang,

255 Kim, \& Mels, 2013). The consequence for misidentifying the number of latent factors results in

256 one of two errors: (a) over-extraction (i.e., extracting and modeling too many factors) and (b)

257 under-extraction (i.e., extracting and modeling too few factors). Over-extraction yields less

258 parsimonious solutions that tend to split meaningful factors into two or more weakly-determined

259 factors (Auerswald \& Moshagen, 2019; Fava \& Velicer, 1992) ${ }^{6}$. Importantly, the detrimental

260 effects of over-extraction are exacerbated as factor loadings and sample sizes decrease (Fava \&

261 Velicer, 1992). In contrast to correlated-factors models - wherein items typically load onto one

262 factor-bifactor models tend to have lower factor loadings because an item's primary loading is

263 bifurcated into loadings on a general factor and one or more group factors. Compared to over-

264 extraction, under-extraction leads to more severely biased factor loadings, which has

\footnotetext{
${ }^{6}$ This effect is demonstrated in Example 2 later in the manuscript, where extracting a third group factor cleaved the 'prudent work-orientation' factor into two separate factors (i.e., prudence and work-orientation).
} 
265 downstream effects, such as distorting the estimated factor scores (Wood, Tataryn, \& Gorsuch, 266 1996).

267 Prior to conducting a factor analysis, it is recommended that researchers jointly consider

268 theoretical perspectives and empirical procedures for determining the number of latent factors to

269 retain (Preacher, Zhang, Kim, \& Mels, 2013). Theoretical insights into a variable domain help

270 decide how to model its structure (e.g., hierarchical versus non-hierarchical) and may even give a

271 plausible range for the number of factors to extract (e.g., five personality factors in the Big Five

272 model of personality; e.g., Digman, 1997). Empirical procedures are data-driven approaches to

273 determine an optimal number of latent factors to model. However, different empirical procedures

274 applied to the same dataset often result in different suggestions - this is exemplified in both

275 datasets later in the manuscript. Moreover, a recent simulation study of dimensionality

276 assessment found that "no single approach displayed the highest accuracy in all conditions"

277 (Auerswald \& Moshagen, 2019, p. 487).

When estimating the dimensionality of a dataset, researchers should seek converging

279 evidence from theoretical insights and multiple empirical procedures (Auerswald \& Moshagen, 280 2019). To estimate the number of factors to model, most methods implement a decision rule 281 based on eigenvalues - properties of the sample-based correlation matrix (e.g., Braeken \& van 282 Assen, 2017). The most popular approach — and the default for many programs, such as SPSS— 283 is to retain all factors associated with eigenvalues greater than one. Although popular, this 284 decision rule has low accuracy and frequently leads to over-extraction (Auerswald \& Moshagan, 285 2019; Cliff, 1988; Hayton, Allen, Scarpello, 2004, Preacher, et al. 2013). A recent and related 286 method relies on the theoretical sampling distributions of eigenvalues to improve the 287 'eigenvalues greater than one' rule. This approach is named the Empirical Kaiser Criterion 
288 (EKC; Braeken \& van Assen, 2017). Aside from EKC, two other methods can accurately detect 289 the correct number of factors to retain in multiple factor models. They are the parallel analysis

290 (PA; Hayton et al., 2004; Horn, 1965) and comparison data (CD; Ruscio \& Roche, 2012)

291 techniques. Briefly, these methods compare sample-based eigenvalues to eigenvalues obtained

292 from computer-generated datasets. Specifically, PA generates random data with no underlying

293 factor model (i.e., a null model) whereas CD generates non-random data with an underlying

294 factor model that is comparable to the sample-based data. In general, if two of these methods

295 (e.g., EKC, PA, and CD) agree on the number of latent factors, there is a good chance they have

296 converged on the correct number of factors (Auerswald \& Moshagen, 2019).

298 in exploratory bifactor analysis, researchers have numerous methodological options at their

299 disposal for estimating a bifactor solution. For simplicity, the competing methods can be

300 distinguished on two dimensions (see Table 1). The first dimension is the analytic strategy used

301 (i.e., how a bifactor pattern is obtained) with categories of (a) hybrid approaches, (b) target

302 rotations, and (c) analytic bifactor rotations. In this context, hybrid approaches are generally

303 conducted in two stages to obtain bifactor parameter estimates. For instance, in the Schmid-

304 Leiman (SL; Schmid \& Leiman, 1957) method, one first conducts a higher-order factor analysis

305 and then re-expresses the higher-order parameter estimates into a constrained (hierarchical)

306 bifactor pattern. Similarly, target rotation methods have applied either partially- (Abad et al.,

307 2017; Browne, 2001) or fully-specified (Waller, 2018) target matrices (i.e., a factor rotation

308 toward a supplied target structure, like a bifactor structure) to obtain a bifactor solution. Lastly,

309 analytic bifactor rotations (Jennrich \& Bentler, 2011, 2012, 2013) can be used to rotate the

310 factors from an exploratory factor analysis directly to a bifactor pattern. 
The second dimension characterizing bifactor estimation methods (Table 1) concerns the

312 type of model that is ultimately obtained. These procedures can be divided into those that

313 estimate either a (constrained) hierarchical or (unconstrained) non-hierarchical bifactor model.

314 Although a thorough discussion of each method and its underlying mechanics is beyond the

315 scope of this manuscript (cf. Abad et al., 2017; Giordano \& Waller, 2020), in what follows we

316 briefly describe the popular approaches - highlighting their benefits and drawbacks — for

317 estimating exploratory bifactor measurement models.

318 To estimate exploratory bifactor measurement models, a prominent analytic strategy is

319 that of analytic rotations. Until recently, no analytic rotations (e.g., varimax, oblimin, promax)

320 were capable of directly estimating a bifactor solution. Recent authors have addressed this gap

321 by extending the quartimin and geomin rotation criteria to recover non-hierarchical (i.e.,

322 unconstrained and full-rank) bifactor models (Jennrich \& Bentler, 2011, 2012, 2013). These

323 rotations are known as the bifactor quartimin and bifactor geomin rotations; These rotations

324 should not be confused with their non-bifactor analogues that are intended to find simple

325 structure in the traditional factor analysis paradigm. Unfortunately, two comprehensive studies

326 have found that bifactor quartimin and bifactor geomin rotations are among the least accurate

327 methods for estimating exploratory bifactor measurement models (Abad et al., 2017; Giordano \&

328 Waller, 2020).

329 The SL procedure — and its modern cognate, the Direct Schmid-Leiman (DSL; Waller,

330 2018) procedure - provides an alternative to estimating a bifactor model by an analytic rotation.

331 The SL and DSL procedures both estimate a hierarchical (i.e., constrained and rank-deficient)

332 bifactor model. Most applications of the SL procedure transform a second-order model with one

333 general factor but there is no theoretical limit to the number of higher-order levels that can be 
334 transformed (Schmid \& Leiman, 1957; Yung, Thissen, \& McLeod, 1999). As a consequence, SL

335 can estimate general factors at different hierarchical levels when a sufficient number of lower-

336 order factors exist to ensure model identification. ${ }^{7}$ For example, solutions with one general factor

337 need at least three lower-order factors to yield an identified second-order solution (Ledermann,

338 1937). Notably, this shortcoming of the SL method is not shared with the DSL procedure

339 because DSL utilizes a target rotation. Thus, if one's data are best represented by two group

340 factors, a DSL approach will become the optimal estimation method because the SL approach

341 will yield biased parameter estimates in the bifactor measurement model.

342 Best Performing Exploratory Bifactor Analysis Methods. Of the available methods to

343 estimate exploratory bifactor measurement models, three methods seem to outperform the rest:

344 (a) SL, (b) DSL, and (c) iterated Schmid-Leiman target rotation (SLi; Abad et al., 2017). In a

345 comprehensive Monte Carlo simulation (Giordano \& Waller, 2020), SL and DSL were best able

346 to recover both hierarchical and non-hierarchical population models. These two methods,

347 however, were not equally accurate in recovering bifactor solutions. In simplified terms,

348 comparing SL versus DSL is akin to the optimal-weight versus unit-weight argument from the

349 multiple regression literature (e.g., Schmidt, 1971). Namely, DSL applies unit weights to obtain

350 a bifactor pattern and thus should be superior to SL in terms of cross-validation accuracy when

351 sample sizes are small (e.g., $n<500)$. Alternatively, SL obtains a hierarchical bifactor pattern

${ }^{7}$ To check whether a sufficient number of variables (e.g., test items, lower-order factors) exist to produce an identified model in exploratory factor analysis, Ledermann's (1937) inequality can be applied. Specifically, let $k \leq$ $\frac{2 p+1-\sqrt{8 p+1}}{2}$ where $k$ is the maximum number of factors that are identified and $p$ is the number of observed variables. This formula is symmetric such that the minimum number of variables, $p$, needed to identify the number of latent factors, $k$, is quantified where $p \geq \frac{2 k+1+\sqrt{8 k+1}}{2}$. See also the 'Ledermann' function in the fungible $\mathrm{R}$ library (Waller, 2019). 
352 through optimal weights and therefore is more accurate than DSL when sample sizes are large

353 (e.g., $n>500)$. SLi, as a method yielding non-hierarchical and unconstrained bifactor patterns,

354 often surpasses SL and DSL in large samples when cross-loadings are present (e.g., Figure 17 of

355 the supplemental materials in Giordano \& Waller, 2020). Nevertheless, a prominent limitation of

356 the SLi method is its notable tendency for finding bifactor patterns that markedly diverge from

357 the true population values - even in large samples (e.g., $n=2,000)$. Taken together, SL should be

358 applied in studies with large sample sizes and DSL should be applied in studies with small

359 sample sizes. SLi can be applied under both conditions but researchers should be aware of its

360 tendency to produce nonsensical solutions with some datasets (see Figures 1 and 2 and the online

361 supplement of Giordano \& Waller, 2020).

362 Limitations of Target Rotations in Exploratory Bifactor Analyses. The generally good

363 performance of target rotations when estimating bifactor models comes with an important

364 caveat - namely, target rotations often find a desired structure (e.g., a bifactor structure)

365 regardless of the data generating model (e.g., Hurley \& Cattell, 1962). In the present context, if

366 the data generating model is an orthogonal factor pattern without a general factor, the DSL (and

367 related methods, such as the Direct Bifactor) method will likely find (erroneously) a bifactor

368 pattern with a general factor. This shortcoming of bifactor target rotations (i.e., DSL) is not

369 shared by the SL approach.

370 Limitations of the Schmid-Leiman method. Whereas target rotation methods for

371 estimating bifactor models can produce misleading results if the data do not adhere to a bifactor

372 structure, the SL method has its own drawback. When estimating the higher-order model within

373 the SL procedure, the factor structure can be obliquely rotated an infinite number of ways

374 without changing the fit of the estimated solution. In the exploratory factor analysis literature this 
375 issue is known as rotational indeterminacy (Mulaik, 2010, ch. 10). Different oblique rotations 376 (cf. Browne, 2001) apply different criteria for finding simple structure (Thurstone, 1947) pattern

377 matrices, and thus, different rotation methods can produce notably different factor correlation

378 matrices. All else being equal, rotations that yield larger factor correlations will find stronger

379 general factor saturations in an SL transformation. To illustrate the practical implications of

380 rotational indeterminacy ${ }^{8}$, we applied 1,001 different oblique rotations (cf. Crawford \&

381 Ferguson, 1970; see also, Browne, 2001) to the dataset from Example 1. Each rotation was

382 plotted against the estimated general factor saturation from an SL procedure (see Figure 2).

383 Simply put, rotations that seek factor loadings patterns in which each variable loads onto as few

384 factors as possible (i.e., minimizing variable complexity) will often fail to recover indicator

385 cross-loadings. Consequently, when estimated cross-loadings are biased towards zero the

386 estimated factor correlations are upwardly biased, as is the estimated general factor saturation.

387 Thus, oblique rotations that do not penalize cross-loadings - such as (non-bifactor) geomin

388 (Hattori, Zhang, \& Preacher, 2017; Yates, 1987)—may be preferred.

\section{Interpreting Exploratory Bifactor Models}

\footnotetext{
${ }^{8}$ Another potential implication of rotational indeterminacy in the SL procedure is the downstream effect on other methods incorporating SL procedures to obtain bifactor measurement models. Specifically, the SLi and SLt methods initiate estimation using starting values obtained from an SL solution. Thus, differences in the SL starting values (i.e., SL solutions from different oblique rotations) may result in differences in the final parameter estimates.
} 
Once a bifactor model is estimated, researchers can begin to interpret the estimated

394 parameters. In this section we briefly discuss how bifactor models can be leveraged to better

395 understand the underlying structure of multidimensional data in two ways. First, in the context of

396 bifactor measurement models (Rodriguez et al., 2016), we describe indices designed to assess the

397 relationships between observed variables (e.g., scale items, homogenous item parcel scores) and

398 latent factors. Second, we introduce difficulties that are unique to bifactor models in relating

399 estimated latent factors to external variables (i.e., using factor scores; Grice, 2001).

$400 \quad$ Relating Latent Factors to Observed Variables. A bifactor measurement model is a

401 useful tool for partitioning variance into uncorrelated latent factors, particularly when modeling

402 multidimensional indicators in a given construct domain (Reise, 2012; Rodriguez et al., 2016).

403 The utility of bifactor models - compared to correlated-factor models - is readily apparent in

404 viewing the estimated factor loadings. All factor loadings estimates are regression coefficients

405 relating the latent factors (i.e., the independent variables) to the observed variables (i.e., the

406 dependent variables). Much like in multiple regression with uncorrelated independent variables,

407 in a bifactor solution with uncorrelated factors, these regression coefficients are equivalent to

408 zero-order correlations (Holzinger, 1937). Moreover, squared factor loadings in a bifactor model

409 (i.e., squared correlation coefficients) represent the proportion of variance in the observed

410 variable that is accounted for by a given factor. Alternatively, in a correlated-factors model, the

411 factor loadings are standardized regression weights (i.e., not zero-order correlations) and must

412 therefore be interpreted as such. In short, factor loadings in bifactor models are simpler to

413 interpret.

414 In orthogonal models, the relation between one variable and one factor is captured by the

415 factor loading. To represent the collective effect of the general and group factors on a given 
416 variable, a researcher can calculate the communality $\left(h^{2}\right)$ for each variable. With standardized

417 factor loadings, communality values reflect the proportion of observed variable variance that is

418 collectively due to the common factors. The remaining variance (captured by the uniqueness

419 factors) is a combination of measurement error and specific factor influences that is not shared

420 with other variables. From the communality values, another closely related index can be

421 calculated to understand the dimensionality of the obtained bifactor solution. Namely, an item's

422 explained common variance (I-ECV) index (Reise et al., 2010; Rodriguez et al., 2016; see also,

423 ten Berge \& Sočan, 2004). In essence, I-ECV represents the proportion of item communality that

424 can be ascribed to the general factor. When examined in tandem, $h^{2}$ and I-ECV values let a

425 researcher see (a) how saturated each item is with group and general factor variance and (b) how

426 much of that latent factor saturation is due to the general factor. In other words, these indices

427 provide useful insight into the dimensionality of each item in a bifactor measurement model. In

428 later sections, we estimate bifactor models in two datasets to illustrate the computation and 429 interpretation of the $h^{2}$ and I-ECV indices.

$430 \quad$ Relating Latent Factors to Observed Scale Variance. Whereas the previous section 431 described various methods to conceptualize the relationship between each observed variable and

432 one or more common factors, this section is concerned with indices that assess how factors

433 account for variance in the summed (standardized) scale scores. To illustrate the difference

434 between these ideas, recall Toker and Ackerman's (2012) examination of complexity interests in

435 STEM students. Whereas $h^{2}$ and I-ECV will quantify factor saturation for any given scale item,

436 we need different indices to quantify factor saturation across item combinations (such as items

437 forming a subscale). These indices represent model-based reliability indices (e.g., Rodriguez et 438 al., 2016; Zinbarg, Revelle, Yovel, \& Li, 2005). Note that we use the term 'model-based 
439 reliability' to differentiate these measures from traditional reliability indices (i.e., the ratio of true 440 score variance to observed score variance). Specifically, model-based reliability focuses on 441 aspects of the true scores that are due to the common factors (for more details, see Rodriguez et 442 al., 2016).

443 One of the more prominent model-based reliability indices that is based on common

444 factor models is called coefficient omega ( $\omega$; McDonald, 1999; Rodriguez et al., 2016; Zinbarg 445 et al., 2005; see also Ferrando \& Lorenzo-Seva, 2018). This index represents the ratio of 446 common factor variance (i.e., aggregated across the general and group factors) to the observed 447 variance of the unit-weighted total score (computed from standardized item scores). Although 448 unit-weighted sum scores are a suboptimal method for estimating factor scores (Grice, 2001;

449 Grice \& Harris, 1998), they are the most commonly applied method for estimating factor scores.

450 Because $\omega$ is interpreted in the context of unit-weighted sum scores, it is therefore well-suited for 451 applications of bifactor measurement models that rely on unit-weighted scores (for a comparable 452 model-based reliability index using optimally-weighted scoring, see Ferrando \& Lorenzo-Seva, 453 2018; Rodriguez et al., 2016).

454 Variations of $\omega$ can also be computed to better understand how individual factors or a 455 combination of factors relate to the sum scores (e.g., reflecting a subscale; cf. Rodriguez et al., 456 2016). For instance, omega hierarchical $\omega_{h}$ reflects the proportion of the total observed score 457 variance that is attributed to the general factor. Thus, the square-root of $\omega_{h}$ represents the 458 correlation between the general-factor factor scores and the observed sum scores (when the item 459 scores have been standardized). Moreover, the ratio of $\omega_{h}$ over $\omega$ indicates how much latent 460 factor variance (i.e., general and group factor variance) is due to the general factor. As this latter 461 value approaches 1.0 (its maximum) the estimated model approaches a unidimensional structure. 
462 Another notable modification to $\omega$ is called $\omega$ hierarchical subscale $\left(\omega_{h s}\right)$. This index represents

463 the unique portion of subscale score variance that is due to the associated group factor.

464 Importantly, when computing $\omega_{h s}$, the bifactor loadings matrix is subset to only include those

465 variables that are included in the subscale of interest. Taken together, $\omega$ and its cognates inform a

466 researcher on the relative strength of factors in relation to (either overall or subscale) observed

467 scores. For a review of these indices, the reader may consult Rodriguez et al. (2016).

Relating Latent Factors to External Variables. If a researcher is interested in relating

469 factors from an exploratory, non-hierarchical bifactor measurement model to an external

470 variable, they must rely on estimated factor scores as imperfect proxies of the true factor scores

471 (Grice, 2001; Grice \& Harris, 1998; Tucker, 1971). Importantly, as demonstrated by Steiger

472 (1979), correlations between true factor scores and an external variable can differ markedly from

473 the associated correlations obtained when using estimated factor scores. Unfortunately, as

474 described more fully below, the use of estimated factor scores from hierarchical bifactor models

475 is fraught with challenging psychometric obstacles.

Estimated factor scores represent an individual's predicted score on each of the modeled

477 factors (e.g., a person's level of general cognitive ability on a cognitive ability test). Importantly,

478 the most pervasive application of estimated factor scores is when a researcher sums all items

479 exhibiting salient loadings on a particular factor (e.g., factor loadings $\geq|.30|$ ). These unit-

480 weighted scores fail to consider that (a) some variables are better indicators of the latent factors

481 than other variables (i.e., differences in their factor scoring weights; Grice, 2001) and (b) some

482 variables are influenced by multiple group factors causing inflated correlations among the factor

483 score estimates (due to the correlated error variance that results from using unit-weighted

484 estimates). Consequently, unit-weighted factor score estimates "may be highly correlated even 
485 when the factors are orthogonal and they will be less valid representations of the factors in 486 comparison with the refined factor scores [e.g., Thurstone's regression-based estimates]" (Grice, 487 2001, p. 434). Unit-weighted sum scores are therefore generally considered poor estimates of 488 factor scores (Grice, 2001; Grice \& Harris, 1998) unless researchers are working with small 489 samples.

491 constrained hierarchical bifactor models. Thus, in this section, we illustrate some problems in 492 estimating factor scores that are unique to constrained (hierarchical) exploratory bifactor models.

493 To understand these problems, it is informative to first consider the difference between an

494 individual's true factor score and their estimated factor score. Theoretically, all individuals have 495 a true standing on all latent factors (e.g., their cognitive ability scores, realistic interest scores, 496 job satisfaction scores), although their exact standing is both unknown and unknowable in 497 research contexts. Consequently, these factor scores must be estimated. Unfortunately, 498 differences between true and estimated factor scores can be large (e.g., when few items define a 499 factor and factor loadings are low; Guttman, 1955). When this occurs, the correlations between 500 the estimated factor scores and external criteria may present a distorted picture of how the true 501 factor scores relate to the external criteria (Steiger, 1979). In bifactor measurement models, estimated and true factor scores can differ in multiple 503 ways. One important divergence occurs in constrained hierarchical bifactor models. Specifically, 504 because the factor loadings in these models are rank-deficient, the estimated factor scores (with 505 the exception of unit-weighted scores; but see Table 4 for cautionary notes on using unit506 weighted scores in hierarchical bifactor models) are also rank-deficient. Moreover, due to this 507 property, some factor score estimates (e.g., ten Berge, Krijnen, Wansbeek, \& Shapiro, 1999) 
508 cannot be calculated. In constrained hierarchical bifactor models, the estimated loadings on any 509 factor (i.e., general or group) can be perfectly predicted from the estimated factor loadings from

510 the remaining factors. Moreover, due to the rank-deficiency of the factor loadings matrix, the

511 estimated factor scores on any factor can be perfectly reproduced from the estimated scores on

512 the remaining factors. This problem of perfect collinearity has two practical ramifications when

513 relating estimated bifactor scores (from constrained, hierarchical models) to external variables.

514 First, due to the constraints in the bifactor loadings pattern, factor scoring methods cannot yield

515 uncorrelated factor score estimates. Thus, although both the constrained (hierarchical) and

516 unconstrained (non-hierarchical) bifactor models are composed of orthogonal factors, the

517 estimated group and general factor scores in the former model will necessarily be correlated.

518 Second, statistical analyses with estimated factor scores (e.g., via Thurstone's or Harman's

519 method; cf. Grice, 2001) from constrained, hierarchical bifactor models may be inestimable due

520 to the multicollinearity of the estimated scores. For example, multiple regression models with

521 estimated factor scores from constrained, hierarchical bifactor models as predictors cannot

522 isolate the unique effects of the (theoretically orthogonal) predictors (i.e., the estimated general

523 and group factor scores) due to the aforementioned rank-deficient property. Non-hierarchical

524 bifactor models do not include these problematic constraints and thus their estimated factor

525 scores will not be collinear (i.e., perfectly correlated) in empirical applications. Note that in

526 (unconstrained) non-hierarchical bifactor models, it is possible (though not always desirable) to

527 compute orthogonal estimated factor scores (e.g., ten Berge et al., 1999; see also, McDonald \& 528 Burr, 1967; Tucker, 1971).

529 Estimated factor scores in all bifactor models have several drawbacks that merit 530 consideration. The most salient of these drawbacks is the problem of factor score indeterminacy 
531 (Guttman, 1955; Steiger, 1979; Wilson, 1928). Simply put, factor score indeterminacy means

532 that factor scores cannot be uniquely calculated, although they can be uniquely estimated

533 (Wilson, 1928). In more simple terms, "for any factor scores...satisfying the factor model, there

534 exists also a different set of factor scores..., which also satisfy the model" (Steiger \&

535 Schönemann, 1978, p.151). In practice, not only are true factor scores unknowable, estimated

536 factor scores from one method can differ from those obtained by another method (Grice \&

537 Harris, 1998). For instance, unit-weighted factor scores can produce notably different estimates

538 than those obtained from other factor score estimation methods (cf. Grice, 2001).

\section{Example Explorations of Bifactor Models in Vocational Behavior}

In the previous section, we reviewed several important decisions (and their

541 consequences) when fitting bifactor models to vocational data. In this section, to exemplify the

542 concepts described previously, we fit constrained bifactor models to two vocational behavior

543 datasets. In the first example, we illustrate a bifactor model of rater effects in the measurement of

544 developmental performance feedback ratings (Hoffman, Lance, Bynum, \& Gentry, 2010). In the

545 second example, we illustrate a bifactor model to better understand the dimensional structure of

546 conscientiousness (e.g., Hogan \& Ones, 1997; Roberts, Chernyshenko, Stark, \& Goldberg,

547 2005).

548 Example 1: Bifactor Modeling Rater Effects in Developmental Performance Feedback

549 Organizations often provide developmental performance feedback to employees using

$550360^{\circ}$ evaluation systems (i.e., collecting ratings of a focal individual from multiple unique

551 perspectives; Craig \& Hannum, 2006). Such ratings are frequently used in employee

552 development efforts (Smither, London, \& Reilly, 2005). Multirater feedback systems rest on the

553 premise that raters from different perspectives provide complementary insights into the 
554 performance of the ratees. Thus, a bifactor model is the perfect vehicle for disentangling a

555 general performance factor from group factors reflecting rater-specific vantage points (e.g.,

556 supervisor, peer, subordinate, and self). To illustrate this idea, we reanalyzed published

557 multisource, developmental ratings of managerial performance (Hoffman, et al., 2010).

558 Sample Description. To provide developmental feedback about a manager's

559 performance, Hoffman and colleagues (2010) obtained data from a multisource performance

560 feedback assessment tool called BENCHMARKS (Lombardo, McCauley, McDonald-Mann, \&

561 Leslie, 1999). Managers were rated on scales measuring three performance dimensions: (a)

562 technical performance, (b) interpersonal performance, and (c) leadership. Ratings were obtained

563 from the following sources: (a) two supervisor ratings, (b) two peer ratings, (c) two subordinate

564 ratings, and (d) self-ratings. In total, 22,420 managers were assessed with a combined total of

565156,940 raters. Hoffman et al. (2010, p. 129-130) described the managerial sample as consisting

566 "primarily of White (76\%) male (64\%) college graduates (88\%)" with an average age of 42 . The

567 BENCHMARKS instrument included 115 items. For these analyses, we used aggregated scale-

568 level data with one rater per source yielding 12 scores ( 4 rater perspectives [sources] $\times 3$ scales

569 [performance dimensions] $=12$ factor indicators). To align our research with applied best

570 practices to minimize interrater measurement error (Ones, Viswesvaran, \& Schmidt, 2008;

571 Viswesvaran, Ones, \& Schmidt, 1996), sources with multiple raters were combined into

572 composites (e.g., both supervisor ratings were composited into a general supervisor rating).

573 Bifactor Modeling. To estimate a bifactor measurement model, we employed a mixture

574 of rational/theoretical and empirical modeling strategies. This approach originates from the

575 contemporary philosophies of factor analysts wherein "model selection is not intended to find the

576 true model but rather is intended to find a parsimonious model that gives reasonable fit" 
577 (Preacher, Zhang, Kim, \& Mels, 2013, p. 52). Empirically, we relied on scree plots (Cattell,

578 1966) and the Empirical Kaiser Criterion (EKC; Braeken \& van Assen) to help identify a

579 plausible number of factors to retain. Both methods suggested the presence of four factors.

580 Among the two types of bifactor models that have been discussed in this manuscript, we fit a

581 constrained (hierarchical) bifactor model as this model is better aligned with the hierarchical

582 relations among the developmental ratings.

584 surmised. All raters assessed managers on the same, highly-correlated performance dimensions

585 (see the online supplement). Thus, the four rating perspectives should be correlated to the extent

586 that they all measure managerial performance. Moreover, each rating may be associated with

587 systematic variance that is unique to each rating source (e.g., rating biases, unique performance

588 insights). These combined influences on managerial performance can be modeled as a second-

589 order factor model with four correlated factors (rating sources). These correlated factors are in

590 turn influenced by a higher-order, general (performance) factor. As described by Schmid and

591 Leiman (1957; see also Thomson, 1951; Thurstone, 1947), one can transform this higher-order

592 model into a constrained, hierarchical bifactor model with a single general factor and four

593 orthogonal group factors, each representing a rating perspective effect. In other words, the

594 correlations among ratings of managerial performance are a function of (a) the manager's true

595 general performance and (b) idiosyncratic perspective effects (e.g., boss or subordinate

596 perspectives). Given the uncharacteristically large sample size for these data, we applied the SL

597 (Schmid \& Leiman, 1957) procedure to generate a constrained, hierarchical bifactor model of 
managerial performance. ${ }^{9}$ To aid in the interpretation of this model, we computed communality

$599\left(h^{2}\right)$ values, I-ECV indices, and several variants of coefficient $\omega$ (McDonald, 1999; Rodriguez et

600 al., 2016; Zinbarg et al., 2005).

601 Results. Table 2 contains the estimated bifactor measurement model for the

602 developmental performance ratings (Hoffman et al., 2010). Note that this model included one

603 general and four group factors. The number of group factors is consistent with the

604 recommendations of the scree and EKC plots. The results shown in Table 2 suggest that these

605 group factors represent perspective effects (i.e., boss, peer, subordinate, and self-rated effects) on

606 the managerial ratings.

As shown in Table 2, the factor loadings $(\lambda)$ on the general performance dimension were

611 substantially lower $(.21 \leq \lambda \leq .59)$ than the primary loadings for the rater-perspective factors:

612 boss ratings $(.76 \leq \lambda \leq .86)$, peer ratings $(.65 \leq \lambda \leq .78)$, subordinate ratings $(.63 \leq \lambda \leq .77)$, and

613 self-ratings $(.78 \leq \lambda \leq .90)$. As expected, there are virtually no cross-loadings present in the

614 estimated bifactor model. Interestingly, ratings by peers and subordinates produced factor

615 loadings on the general performance factor $(.51 \leq \lambda \leq .59)$ that were systematically larger than

616 those generated by either the boss $(.44 \leq \lambda \leq .48)$ or self-report ratings $(.21 \leq \lambda \leq .28)$. Moreover,

617 within each rating perspective, there was a consistent trend in relative factor loading sizes:

\footnotetext{
${ }^{9}$ When estimating the constrained bifactor model, we extracted unweighted (ordinary) least squares factor loadings. The first-order factor solution was subsequently rotated using an oblique geomin rotation from 100 random starting configurations (cf. Rozeboom, 1992) and a geomin tuning parameter set to .01 (cf. Hattori et al., 2017).
} 
618 technical performance $>$ interpersonal performance $>$ leadership. Here, the reader should recall

619 that bifactor loadings can be interpreted as correlations. Therefore, technical performance ratings

620 are more highly correlated with both the general performance factor and the rater-perspective

621 effects than interpersonal and leadership performance behaviors. Leadership ratings were the

622 least highly correlated with the general performance factor.

623 The two right-most columns of Table 2 display the communalities and the I-ECV values.

624 Communalities for the factor indicators ranged from $.66 \leq h^{2} \leq .97$, meaning that, collectively,

625 the latent factors accounted for between $66 \%$ to $97 \%$ of the observed indicator variance. I-ECV

626 values ranged from $.07 \leq \mathrm{I}-\mathrm{ECV} \leq .40$. Thus, $7 \%$ to $40 \%$ of the reliable performance ratings

627 variance was attributed to general performance with the remaining $60 \%$ or more due to

628 perspective effects. Moreover, I-ECV values suggested that self-ratings $(.07 \leq \mathrm{I}-\mathrm{ECV} \leq .11)$ were

629 prominently lower in general (performance) factor saturation than boss $(.24 \leq \mathrm{I}-\mathrm{ECV} \leq .27)$, peer

$630(.36 \leq \mathrm{I}-\mathrm{ECV} \leq .40)$, and subordinate $(.36 \leq \mathrm{I}-\mathrm{ECV} \leq .40)$ ratings. Although factor scores were

631 not (and could not be) computed in this dataset, Guttman's (1955) factor determinacy index $(\rho)$

632 was computed for each factor. The general factor was less determinant $(\rho=.78)$ than the boss

633 (.92), peer (.86), subordinate (.86) and self-ratings (.95).

634 For the model reported in Table 2, The coefficient $\omega$ model-based reliability index was

635 high $(\omega=.96)$, suggesting that the general and group factors collectively accounted for about

$63696 \%$ of the (unit-weighted) sum score variance. Moreover, the general performance factor alone

637 accounted for $56 \%\left(\omega_{h}=.56\right)$ of the sum score variance. Taken together, the general

638 performance factor represents the majority (58\%) of all common factor variance (i.e., the ratio of

$639 \omega$ to $\omega_{h}$ ) and the rater-perspective effects (i.e., the group factors) accounted for the remaining

$640(42 \%)$ common factor variance. 
When partitioning variance at the subscale level (i.e., $\omega_{h s}$ ), group factors associated with

642 the boss, peer, subordinate, and self-report perspectives each accounted for $73 \%, 60 \%, 59 \%$, and

$64384 \%$ of the variance, respectively. Simply put, performance ratings from any one perspective are

644 predominately unrelated to general (overall) managerial performance. Specifically, ratings from

645 bosses, peers, subordinates, and the self only share $27 \%, 40 \%, 41 \%$, and $16 \%$ (respectively) of

646 their variance with the general performance factor. Comparing $\omega_{h}$ to $\omega_{h s}$ highlights the utility of

647 multisource feedback ratings. Namely, ratings from any one perspective are unreliable and

648 therefore insufficient to assess overall managerial performance. Nevertheless, reliability of

649 performance ratings quickly increases as more perspectives are combined together.

650 Implications. Our re-analyses of the Hoffman et al. (2010) performance evaluations

651 provided novel insights into single-source versus multiple-source ratings of managerial

652 performance. Specifically, our $\omega_{h s}$ analyses demonstrated the relative contributions of rater

653 perspectives on the overall observed variance. These results suggest that a substantial $84 \%$ of the

654 observed variance in self-reported performance ratings is unrelated to the general performance

655 factor. In contrast, across our modeled rating perspectives, results suggest that subordinate raters

656 (followed closely by peer raters) have the lowest perspective-specific effects (59\%). Subordinate

657 ratings of performance are less contaminated with source-perspective effects and have among the

658 highest correlations (i.e., factor loadings) with the general performance factor. Moreover,

659 subordinate and peer managerial performance ratings are more strongly influenced by the general

660 performance factor (i.e., higher I-ECV values) than either boss or self-reported perspectives.

661 These results imply that subordinate and peer raters are the best single-source raters of

662 managerial performance for developmental purposes. 
In summary, although each rating source of managerial performance is predominately

664 influenced by perspective-specific effects, the results of our (constrained) bifactor analysis

665 suggests that a general performance factor accounted for the lion's share $(56 \%)$ of variance in the

666 collective multisource feedback ratings. This latter finding is novel to the present article.

667 Hoffman et al. (2010) reported a variance accounted for index for each rating perspective and

668 averaged across these values to summarize their results. They found that, on average, a general

669 performance factor accounted for $3 \%$ of the variance in their models. However, averaging across

670 raters fails to consider prominent psychometric concepts. Namely, that when combining parallel

671 assessments of the same constructs, true score variance accumulates faster than error score

672 variance. In this vein, a grand mean will appreciably underrepresent the overall general

673 performance factor saturation across parallel assessments compared to the present findings that

674 are based on the full bifactor model. In practice, the differences between the present findings and

675 those of the published findings translate into different recommendations about multisource

676 developmental ratings. The small grand mean value reported by Hoffman et al. (2010) suggests

677 that multisource ratings are an expensive and inefficient undertaking. However, our resulting

678 bifactor analyses suggest that multiple rating sources provide developmentally informative and

679 more accurate insights into employee performance.

680 Example 2: Bifactor Modeling of Conscientiousness Inventories

681 Conscientiousness is a potent predictor of workplace behaviors and outcomes (Roberts et 682 al., 2005; Wilmot \& Ones, 2019). Moreover, it is perhaps the best personality determinant of 683 training and educational performance (Connelly \& Ones, 2010; Poropat, 2009). Furthermore, 684 conscientiousness has been implicated as a determinant of satisfaction and well-being at work 
685 (Seltzer, Ones, \& Tatar, 2017) and health more generally (Bogg \& Roberts, 2004). Thus, the impact of conscientiousness on vocational preparation and performance is notable.

At its core, conscientiousness refers to a person's tendency to "follow rules and prioritize non-immediate goals" (DeYoung, 2015, p. 45). Individuals high in conscientiousness are often described as hardworking, orderly, responsible, self-controlled, and rule-abiding (Stanek \& Ones, 2018). Of relevance for the present manuscript, conscientiousness is also a multidimensional construct (Hogan \& Ones, 1997; Stanek \& Ones, 2018). A number of

692 empirical studies have sought to identify its lower level structure (e.g., DeYoung, Quilty, \& 693 Peterson, 2007; Roberts et al., 2005) though, currently, there is no consensus on the number and 694 nature of the lower order traits (Roberts, Lejuez, Krueger, Richards, \& Hill, 2014). Along this 695 vein, we estimated an exploratory bifactor model of 11 conscientiousness facet scales to 696 elucidate the dimensional structure of this domain. We use these data to illustrate aforementioned 697 problems that can arise when estimating factor scores for constrained (hierarchical) bifactor 698 models.

Sample and Data Description. Conscientiousness facet scales were administered to 761 undergraduate students at a large, Midwestern university. Participants were recruited online

701 through the University's research participant pool. Participants completed the entire study online.

702 The sample was fairly typical for a Midwestern collegiate sample and was primarily composed 703 of White $(75.0 \%)$ females $(68.2 \%)$ with an average age of $(21.0, S D=2.9)$. The remaining 704 participants identified as Asian/Pacific Islander (13.0\%), multi-racial (4.5\%), Black (3.4\%), or 705 Hispanic/Latino (2.6\%). In order to represent conscientiousness facets (i.e., subdimensions) that have appeared in 707 various conceptualizations of this domain (e.g., Roberts et al., 2005), multiple scales assessing 
708 all known facets of conscientiousness were administered to the sample. Eleven conscientiousness 709 facet scales - achievement striving, cautiousness, dutifulness, industriousness, orderliness,

710 persistence, responsibility, traditionalism, and virtue - were selected from the International

711 Personality Item Pool according to the work of Hough and Ones (2002), Roberts et al. (2005),

712 and Stanek and Ones (2018) to form a content valid representation of the conscientiousness

713 facets. Participants rated how accurately each item described them on a five-point scale $(1=$

714 "Very Inaccurate" to 5 = "Very Accurate"). Attention checks were used, and careless responders

715 were excluded from analyses.

716 Bifactor Modeling. A series of hierarchical bifactor models were applied to evaluate the

717 structure of conscientiousness. Prior to performing these analyses, we ran several preliminary

718 analyses (i.e., scree and EKC plots) to determine the latent dimensionality underlying the data.

719 We also considered prior theoretical work in this domain to decide on the optimal number of

720 group factors to include in the bifactor model. Prior work (over several decades) has supported

721 views (e.g., Digman, 1997) about the hierarchical nature of conscientiousness (Stanek \& Ones,

722 2018). Most recently, DeYoung and colleagues (DeYoung, 2015; DeYoung, Quilty, \& Peterson,

723 2007) have presented empirical and theoretical support for two subdimensions of

724 conscientiousness: orderliness and industriousness. These lower-order factors encompass various

725 facets of conscientiousness that are influenced by a general conscientiousness factor. Once a

726 constrained bifactor model was estimated, factor scores were estimated for all 761 students.

727 Although in hierarchical bifactor models the estimated factor scores are not linearly

728 independent - meaning that the estimated scores on one factor can be perfectly reproduced from

729 the estimated scores on the remaining factors - for didactic purposes, we estimated factor scores

730 for this example. Specifically, we estimated: (a) unit-weighted factor scores, more commonly 
731 known as sum scores, and (b) Thurstone's (1947) regression-based factor scores (Grice, 2001;

732 McDonald \& Burr, 1967; Tucker, 1971).

733 Results. Table 3 contains the estimated bifactor measurement model of the 11

734 conscientiousness subscales. Scree and EKC plots jointly recommended the extraction of three

735 factors. However, prior theory strongly suggested that two factors are best able to explain

736 variation in the lower-order conscientiousness factors (DeYoung, 2015). Thus, two constrained

737 bifactor models were estimated, a DSL bifactor model with two group factors and an SL bifactor

738 model with three group factors. ${ }^{10}$ Both models, subjectively speaking, were equally interpretable.

739 However, in the three-group-factor solution, the first group factor (and the items loading onto it)

740 was cleaved in two. This produced two weakly-determined group factors, each marked by only

741 two observed variables. Thus, the theoretically supported, two-group-factor DSL solution was

742 retained (see Table 3). Interested readers can consult the online supplement to see the

743 conscientiousness bifactor model with three group factors.

Insert Table 3 here

In the conscientiousness bifactor measurement model, factor loadings $(\lambda)$ on the general

748 conscientiousness factor ranged from small to moderately large $(.29 \leq \lambda \leq .58)$. Using a common,

749 through arbitrary, cutoff to identify which items saliently load onto each factor (i.e., $\lambda \geq|.30|$ ),

\footnotetext{
${ }^{10}$ An SL procedure is inappropriate in cases where fewer than three group factors are present. In a (pre-transformed) higher-order model, the higher-order factor must influence at least three first-order factors to uniquely determine the factor loadings. If two lower-order factors are present, factor loadings on the higher-order factor will be biased which, in turn, will bias the SL bifactor loadings parameters. A DSL procedure directly estimates a constrained bifactor model without first conducting a higher-order factor model and therefore does not suffer from these biases.
} 
750 the conscientiousness subscales could be categorized under the two group factors. The first group

751 factor was related to the following subscales (with salient group factor loadings in parentheses):

752 diligence $(\lambda=.70)$, achievement $(\lambda=.67)$, persistence $(\lambda=.64)$, industriousness $(\lambda=.44)$, virtue

$753(\lambda=.43)$, deliberateness $(\lambda=.41)$, and cautiousness $(\lambda=.31)$. We interpreted this group factor as

754 prudent work orientation. The second group factor was related to the following subscales:

755 dutifulness $(\lambda=.83)$, traditionalism $(\lambda=.40)$, and responsibility $(\lambda=.36)$. We interpreted this

756 group factor as conformity. Interestingly, in this sample, the orderliness scale had relatively weak

757 loadings on all factors, though its largest loading was on the general conscientiousness factor $(\lambda$

$758=.37)$, with weaker loadings on both the first $(\lambda=.29)$ and second $(\lambda=.22)$ group factors. ${ }^{11}$

759 The subscales varied greatly in how variance was partitioned across the factors.

760 Communalities ranged considerably $\left(.25 \leq h^{2} \leq .99\right)$ with the dutifulness $\left(h^{2}=.99\right)$ subscale

761 being almost entirely comprised of latent factor variance (i.e., general conscientiousness variance

762 and conformity group factor variance). Alternatively, orderliness $\left(h^{2}=.27\right)$ and traditionalism

$763\left(h^{2}=.25\right)$ shared less than $30 \%$ of their observed variance with the three latent

764 conscientiousness factors. This suggests that for both orderliness and traditionalism, there may

765 be other latent personality factors (e.g., neuroticism for orderliness, and openness for

766 traditionalism) accounting for reliable variance beyond conscientiousness. For example,

767 Connelly and colleagues (2014) found that traditionalism is related to both low openness and

768 high conscientiousness.

${ }^{11}$ In the conscientiousness bifactor model with three group factors, the prudent work orientation factor was bifurcated into two factors: prudence and work orientation. The latter work orientation factor appears to be fully in line with that industriousness aspect proposed by DeYoung (2015) and colleagues (DeYoung et al., 2007). 
Turning to the I-ECV index, the 11 conscientiousness subscales had a somewhat narrow

770 range in their general factor saturation $(.31 \leq \mathrm{I}-\mathrm{ECV} \leq .52)$. Specifically, of the common factor

771 variance, the general conscientiousness factor accounted for $41 \%$ (diligence), $40 \%$

772 (achievement), 42\% (persistence), 42\% (industriousness), 51\% (virtue), 49\% (deliberateness),

$77352 \%$ (cautiousness), 52\% (orderliness), 31\% (dutifulness), 34\% (traditionalism), and 49\%

774 (responsibility) of the various conscientiousness facet scales. Note that these I-ECV values must

775 be considered in conjunction with the communality values. For example, the general

776 conscientiousness factor only accounted for roughly $14 \%$ of the observed variance in the

777 orderliness scale (i.e., $52 \%$ of $27 \%$ ).

778 The estimated conscientiousness bifactor model with two group factors accounted for

$77990 \%$ of the observed total variance $(\omega=.90)$. The general conscientiousness factor accounted for

780 nearly half of all observed variance $\left(\omega_{h}=.46\right)$ but over half $(51 \%)$ of the latent factor variance

781 (i.e., the ratio of $\omega_{h}$ to $\left.\omega\right)$. At the subscale level (i.e., $\omega_{h s}$ ), the first (prudent work orientation)

782 and second (conformity) group factors each accounted for roughly $37 \%$ and $54 \%$ of the observed

783 subscale variance, respectively.

784 To illustrate problems associated with estimated factor scores in constrained bifactor

785 models, we estimated factor scores for the conscientiousness data using the unit-weighted and

786 Thurstone's (1947) regression-based scoring methods for the 761 subjects. Table 4 contains the

787 correlations between (a) unit-weighted factor score estimates, (b) regression-based factor score

788 estimates, and (c) estimated scores on a given factor across the two estimation methods. Both

789 methods produced highly intercorrelated factor score estimates but the correlations between

790 estimated factor scores were notably higher (in absolute value) for the unit-weighted estimates

791 than the regression-based estimates. Namely, for the unit-weighted estimates, the general factor 
792 scores correlated $r=.97$ and .67 with the first and second group factors, respectively, and the 793 estimated factor scores for the two group factors intercorrelated $r=.51$. Recall that the general 794 and group factors in this model are orthogonal, so observed correlations of .97 and .67 between 795 the general and group factors are highly biased. The regression-based factor score estimates of 796 the general factor correlated $r=.63$ and .52 with prudent work orientation and conformity group

797 factors (respectively) whereas these group factor score estimates were negatively correlated $(r=$ 798 -.34). Across the estimation methods, factor scores were highly_but not perfectly_correlated.

799 Thus, particularly for the group factors, estimated factor scores from one method can appreciably 800 differ from estimates from another method. We remind the reader, however, that no factor

801 scoring method is fully appropriate for the hierarchical bifactor model due to the aforementioned 802 constraints on its factor loadings.

803

Implications. Applying a constrained bifactor model to 11 conscientiousness subscales

807 provided insights into the dimensional structure of conscientiousness. From the obtained bifactor

808 loadings matrix, it is apparent that several scales described as conscientiousness facets are only

809 moderately correlated with the general conscientiousness factor. Particularly, as indicated by its

810 communality, only $27 \%$ of the variance in the orderliness scale is related to conscientiousness

811 and its subdimensions of prudent work orientation and conformity. Moreover, some subscales

812 (e.g., dutifulness) predominately measure group factor variance. In practical terms, this implies

813 that administering a diligence subscale will yield scores mostly reflecting a general

814 conscientiousness factor whereas a dutifulness subscale will yield scores mostly reflecting the 
815 conformity subdimension of conscientiousness. Importantly, not all conscientiousness subscales

816 are exchangeable.

817 Based on theoretical perspectives from the extant literature, we can begin to describe the

818 content domain from the resulting conscientiousness dimensional structure. Namely, the

819 conscientiousness general factor appears to reflect the tendency for people to prioritize long-term

820 goals over immediate gratification (see also Connelly, Ones, Hülsheger, 2018; DeYoung, 2015).

821 The group factor that we labeled prudent work orientation is further distinguished from general

822 conscientiousness by the diligent effort directed to achieving goals, and it roughly corresponds to

823 the industriousness aspect of conscientiousness, with an added element cautiousness (Connelly et

824 al., 2018; DeYoung, 2015). Conformity emerged as the second group factor in our bifactor

825 analyses of the 11 conscientiousness facet scales. This factor uniquely focuses on maintenance of

826 social order, a socially-directed orderliness factor that helps protect long-term goals. Taken

827 together, these group factors appear to reflect the two defining characteristics of

828 conscientiousness. Namely, a person's tendency to "follow rules [conformity] and prioritize non-

829 immediate goals [prudent work orientation]” (DeYoung, 2015, p. 45).

830 The estimated factor scores from the constrained conscientiousness bifactor model

831 illustrate an important shortcoming of this type of model. Specifically, the use of sum scores (or

832 other factor scoring estimators) as estimated factor scores can produce highly misleading results.

833 Notice in Table 4 that, when estimating factor scores via unit-weighted sum scores, the general

834 conscientiousness estimated factor scores are (slightly) more highly correlated with the prudent

835 work orientation estimated factor scores $(r=.97)$ than to the regression-based estimates of the

836 general conscientiousness factor $(r=.96)$. Thus, if a researcher estimates subjects' standings on,

837 say, prudent work orientation via sum scores, they would be incorrect to claim that these scores 
838 are orthogonal to the general conscientiousness factor-despite the researcher estimating an

839 orthogonal bifactor model!

841 In this manuscript, we provided vocational behavior researchers a brief overview of both

842 hierarchical and non-hierarchical exploratory bifactor measurement models. We highlighted

843 potential uses of exploratory bifactor models in vocational psychology, we described the best

844 practices (and the statistical code to implement these practices) for estimating and interpreting

845 bifactor models, and we illustrated these concepts in real-world examples of innovative and

846 useful applications of hierarchical bifactor models. Along the way, we also noted important

847 caveats and areas for future research. In short, we believe that exploratory bifactor models, when

848 appropriately applied, hold great promise for aiding vocational behavior researchers in more

849 clearly disentangling multidimensional sources of variance to better understand their research

850 questions. 


\section{References}

852 Abad, F. J., Garcia-Garzon, E., Garrido, L. E., \& Barrada, J. R. (2017). Iteration of partially specified target matrices: Application to the bi-factor case. Multivariate Behavioral Research, 52(4), 416-429. https://doi.org/10.1080/00273171.2017.1301244

Arthur, M. B., Khapova, S. N., \& Wilderom, C. P. (2005). Career success in a boundaryless career world. Journal of Organizational Behavior, 26(2), 177-202. https://doi.org/10.1002/job.290

Auerswald, M., \& Moshagen, M. (2019). How to determine the number of factors to retain in exploratory factor analysis: A comparison of extraction methods under realistic conditions. Psychological Methods, 24(4), 468-491. https://doi.org/10.1037/met0000200

Beaujean, A. A. (2015). John Carroll's views on intelligence: Bi-factor vs. higher-order models. Journal of Intelligence, 3(4), 121-136. https://doi.org/10.3390/jintelligence3040121

Bogg, T., \& Roberts, B. W. (2004). Conscientiousness and health-related behaviors: a metaanalysis of the leading behavioral contributors to mortality. Psychological Bulletin, 130(6), 887. https://doi.org/10.1037/0033-2909.130.6.887

Browne, M. W. (2001). An overview of analytic rotation in exploratory factor analysis. Multivariate Behavioral Research, 36(1), 111-150. https://doi.org/10.1207/s15327906mbr3601_05

Campbell, D. P., \& Holland, J. L. (1972). A merger in vocational interest research: Applying Holland's theory to Strong's data. Journal of Vocational behavior, 2(4), 353-376. https://doi.org/10.1016/0001-8791(72)90012-7 
874 Campbell, J. P., \& Wiernik, B. M. (2015). The modeling and assessment of work performance. Annual Review of Organizational Psychology and Organizational Behavior, 2, 47-74. https://doi.org/10.1146/annurev-orgpsych-032414-111427

877 Carroll, J. B. (1993). Human cognitive abilities: A survey of factor-analytic studies. Cambridge University Press.

Cattell, R. B. (1966). The Scree test for the number of factors. Multivariate Behavioral Research, 1(2), 245-276. https://doi.org/10.1207/s15327906mbr0102_10

Chen, F. F., West, S., \& Sousa, K. (2006). A comparison of bifactor and second-order models of quality of life. Multivariate Behavioral Research, 41(2), 189-225. https://doi.org/10.1207/s15327906mbr4102_5

884 Cliff, N. (1988). The eigenvalues-greater-than-one rule and the reliability of components. Psychological Bulletin, 103(2), 276--279. https://doi.org/10.1037/0033-2909.103.2.276

Connelly, B. S., \& Ones, D. S. (2010). An other perspective on personality: Meta-analytic integration of observers' accuracy and predictive validity. Psychological bulletin, 136(6),

889 Connelly, B. S., Ones, D. S., Davies, S. E., \& Birkland, A. (2014). Opening up openness: A 1092. https://doi.org/10.1037/a0021212 organizational psychology: theory, measurement and application. In D. S. Ones, N. Anderson \& C. Viswesvaran (Eds.) The sage handbook of industrial, work and 
Exploratory Bifactor Models in Vocational Behavior

organizational psychology (pp. 320-365). 55 City Road, London: SAGE Publications Ltd https://doi.org/10.4135/9781473914940.n13

Craig, S. B., \& Hannum, K. (2006). Research update: 360-degree performance assessment. Consulting Psychology Journal: Practice and Research, 58(2), 117. https://doi.org/10.1037/1065-9293.58.2.117

Crawford, C. B. \& Ferguson, G. A. (1970). A general rotation criterion and its use in orthogonal rotation. Psychometrika, 35(321). https://doi.org/10.1007/bf02310792

Cucina, J., \& Byle, K. (2017). The bifactor model fits better than the higher-order model in more than $90 \%$ of comparisons for mental abilities test batteries. Journal of Intelligence, 5(3), 27. https://doi.org/10.3390/jintelligence5030027

Demerouti, E., Bakker, A. B., Vardakou, I., \& Kantas, A. (2003). The convergent validity of two burnout instruments: A multitrait-multimethod analysis. European Journal of Psychological Assessment, 19(1), 12. https://doi.org/10.1027//1015-5759.19.1.12

DeYoung, C. G., Quilty, L. C., \& Peterson, J. B. (2007). Between facets and domains: 10 aspects of the Big Five. Journal of Personality and Social Psychology, 93(5), 880. https://doi.org/10.1037/0022-3514.93.5.880

DeYoung, C. G. (2015). Cybernetic Big Five theory. Journal of Research in Personality, 56, 3358. https://doi.org/10.1016/j.jrp.2014.07.004

916 Di Fabio, A., \& Kenny, M. E. (2019). Decent work in Italy: Context, conceptualization, and assessment. Journal of Vocational Behavior, 110, 131-143. 
919 Duffy, R. D., Blustein, D. L., Diemer, M. A., \& Autin, K. L. (2016). The psychology of working 920 theory. Journal of Counseling Psychology, 63(2), 127.

$921 \quad$ https://doi.org/10.1037/cou0000140

922 Fava, J. L., \& Velicer, W. F. (1992). The effects of overextraction on factor and component analysis. Multivariate Behavioral Research, 27(3), 387-415.

Ferrando, P. J. \& Lorenzo-Seva, U. (2018). Assessing the quality and appropriateness of factor solutions and factor score estimates in exploratory item factor analysis. Educational and Psychological Measurement, 78(5), 762-780. https://doi.org/10.1177/0013164417719308

Ferreira, J. A., Haase, R. F., Santos, E. R., Rabaça, J. A., Figueiredo, L., Hemami, H. G., \&

Fugate, M., Kinicki, A. J., \& Ashforth, B. E. (2004). Employability: A psycho-social construct, Almeida, L. M. (2019). Decent work in Portugal: Context, conceptualization, and assessment. Journal of Vocational Behavior, 112, 77-91. https://doi.org/10.1016/j.jvb.2019.01.009

Gaudron, J. P., \& Vautier, S. (2007). Analyzing individual differences in vocational, leisure, and family interests: A multitrait-multimethod approach. Journal of Vocational Behavior, 70(3), 561-573. https://doi.org/10.1016/j.jvb.2007.01.004

938 Gignac, G. E. (2008). Higher-order models versus direct hierarchical models: G as superordinate or breadth factor? Psychological Science Quarterly, 50, 21-43. 
940 Gignac, G. E. (2016). The higher-order model imposes a proportionality constraint: That is why

941 the bifactor model tends to fit better. Intelligence, 55, 57-68.

942 http://dx.doi.org/10.1016/j.intell.2016.01.006

943 Giordano, C., \& Waller, N. G. (2020). Recovery of bifactor structures: A comparison of seven methods. Psychological Methods, 25(2), 143-156. https://doi.org/10.1037/met0000227

945 Greene, A. L., Eaton, N. R., Li, K., Forbes, M. K., Krueger, R. F., Markon, K. E., ... Kotov, R. (2019). Are fit indices used to test psychopathology structure biased? A simulation study. Journal of Abnormal Psychology, 128(7), 740-764. http://dx.doi.org/10.1037/abn0000434

Grice, J. W. (2001). Computing and evaluating factor scores. Psychological Methods, 6(4), 430. https://doi.org/10.1037/1082-989X.6.4.430

Grice, J. W., \& Harris, R. J. (1998). A comparison of regression and loading weights for the computation of factor scores. Multivariate Behavioral Research, 33(2), 221-247. https://doi.org/10.1207/s15327906mbr3302__

Guttman, L. (1955). The determinacy of factor score matrices with implications for five other basic problems of common-factor theory. British Journal of Statistical Psychology, 8(2),

Hayton, J. C., Allen, D. G., \& Scarpello, V. (2004). Factor retention decisions in exploratory 65-81. https://doi.org/10.1111/j.2044-8317.1955.tb00321.x

Hattori, M., Zhang, G., \& Preacher, K. J. (2017). Multiple local solutions and geomin rotation. factor analysis: A tutorial on parallel analysis. Organizational Research Methods, 7(2), 191-205. https://doi.org/10.1177/1094428104263675 
962 Hogan, J., \& Ones, D. S. (1997). Conscientiousness and integrity at work In R. Hogan, J.

963

964

965

966

967

968

969

970

971

972

973

974

975

976

977

978

979

980

981

982

983
Johnson, \& S. Briggs (Eds.) Handbook of Personality Psychology (pp. 849-870).

Academic Press. https://doi.org/10.1016/b978-012134645-4/50033-0

Hoffman, B., Lance, C. E., Bynum, B., \& Gentry, W. A. (2010). Rater source effects are alive and well after all. Personnel Psychology, 63(1), 119-151. https://doi.org/10.1111/j.17446570.2009.01164.x

Horn, J. L. (1965). A rationale and test for the number of factors in factor analysis. Psychometrika, 30(2), 179-185. https://doi.org/10.1007/bf02289447

Holzinger, K. J. (1935). Introduction to bi-factor theory; solid and hollow staircase patterns for sets of data from mooseheart (No. 5). University of Chicago.

Holzinger, K. J. (1936). Recent research on unitary mental traits. Journal of Personality, 4(4), 335-343. https://doi.org/10.1111/j.1467-6494.1936.tb02038.x

Holzinger, K. J. (1937). Student manual of factor analysis. University of Chicago Press.

Holzinger, K. J., \& Swineford, F. (1937). The Bi-factor method. Psychometrika, 2(1), 41-54. $\underline{10.1007 / \mathrm{BF} 02287965}$

Holzinger, K. J. (1945). Spearman as I knew him. Psychometrika, 10(4), 231-235. https://doi.org/ 10.1007/BF02288890

Hough, L. M., \& Ones, D. S. (2002). The structure, measurement, validity, and use of personality variables in industrial, work, and organizational psychology. In N. Anderson, D. S. Ones, H. K. Sinangil, \& C. Viswesvaran (Eds.), Handbook of Industrial, Work and Organizational Psychology, Vol. 1. Personnel psychology (pp. 233-277). Thousand Oaks, CA: Sage Publications Ltd. https://doi.org/10.4135/9781848608320.n13 
984 Hurley, J. R., \& Cattell, R. B. (1962). The Procrustes program: Producing direct rotation to test a

985

986

987

988

989

990

991

992

993

994

995

996

997

998

999

1000

1001

1002

1003

1004

1005

1006 hypothesized factor structure. Behavioral Science, 7(2), 258-262. https://doi.org/10.1002/bs.3830070216

Jennrich, R. I., \& Bentler, P. M. (2011). Exploratory bi-factor analysis. Psychometrika, 76(4), 537-549. https://doi.org/10.1007/s11336-011-9218-4

Jennrich, R. I., \& Bentler, P. M. (2012). Exploratory bi-factor analysis: The oblique case. Psychometrika, 77(3), 442-454. https://doi.org/10.1007/s11336-012-9269-1

Jennrich, R. I., \& Bentler, P. M. (2013). Erratum to: Exploratory bi-factor analysis. Psychometrika, 78(3), 556-556. https://doi.org/10.1007/s11336-013-9346-0

Judge, T. A., \& Piccolo, R. F. (2004). Transformational and transactional leadership: a metaanalytic test of their relative validity. Journal of Applied Psychology, 89(5), 755. https://doi.org/10.1037/0021-9010.89.5.755

Karasek Jr, R. A. (1979). Job demands, job decision latitude, and mental strain: Implications for job redesign. Administrative Science Quarterly, 24(2), 285-308. https://doi.org/10.2307/2392498

Ledermann, W. (1937). On the rank of the reduced correlational matrix in multiple-factor analysis. Psychometrika, 2(2), 85-93. https://doi.org/10.1007/bf02288062

Levin, J. (1973) Bifactor analysis of a multitrait-multimethod matrix of leadership criteria in small groups. The Journal of Social Psychology, 89(2), 295-299. https://doi.org/10.1080/00224545.1973.9922602

LePine, J. A., Erez, A., \& Johnson, D. E. (2002). The nature and dimensionality of organizational citizenship behavior: a critical review and meta-analysis. Journal of Applied Psychology, 87(1), 52. https://doi.org/10.1037//0021-9010.87.1.52 
1007 Locke, E. A. (1969). What is job satisfaction? Organizational Behavior and Human

1008 Performance, 4(4), 309-336. https://doi.org/10.1016/0030-5073(69)90013-0

1009 Lombardo, M. M., McCauley, C. D., McDonald-Mann, D., \& Leslie, J. B. (1999).

1010 BENCHMARKS® developmental reference points. Greensboro, NC: Center for Creative

$1011 \quad$ Leadership.

1012 Lorenzo-Seva, U., \& ten Berge, J. M. (2006). Tucker's congruence coefficient as a meaningful

1013 index of factor similarity. Methodology, 2(2), 57-64. https://doi.org/10.1027/1614-

$1014 \quad \underline{2241.2 .2 .57}$

1015 McAbee, S. T., \& Connelly, B. S. (2016). A multi-rater framework for studying personality: The

1016 Trait-Reputation-Identity Model. Psychological Review, 123, 569-591.

1017 https://doi.org/10.1037/rev0000035

1018 McCrae, R. R., \& Costa Jr, P. T. (2004). A contemplated revision of the NEO Five-Factor

1019 Inventory. Personality and Individual Differences, 36(3), 587-596.

$1020 \quad$ https://doi.org/10.1016/s0191-8869(03)00118-1

1021 McDonald, R. P. (1999). Test theory: A unified treatment. Taylor \& Francis.

1022 McDonald, R. P., \& Burr, E. (1967). A comparison of four methods of constructing factor

1023 scores. Psychometrika, 32(4), 381-401. https://doi.org/10.1007/bf02289653

1024 McGrew, K. S. (2005). The Cattell-Horn-Carroll Theory of Cognitive Abilities: Past, Present, 1025 and Future. In D. P. Flanagan \& P. L. Harrison (Eds.), Contemporary Intellectual 1026 Assessment: Theories, Tests, and Issues, 136-181. The Guilford Press.

1027 Morris, J. A., \& Feldman, D. C. (1996). The dimensions, antecedents, and consequences of 1028 emotional labor. Academy of Management Review, 21(4), 986-1010.

1029 https://doi.org/10.5465/amr.1996.9704071861 
1030 Mulaik, S. A. (2010). Foundations of Factor Analysis. Boca Raton, FL: CRC Press.

1031 Mulaik, S. A., \& Quartetti, D. A. (1997). First order or higher order general factor? Structural

1032 Equation Modeling: A Multidisciplinary Journal, 4(3), 193-211. https://doi.org/10.1080/10705519709540071

1034 Murray, A. L., \& Johnson, W. (2013). The limitations of model fit in comparing the bi-factor versus higher-order models of human cognitive ability structure. Intelligence, 41(5), 407422. https://doi.org/10.1016/j.intell.2013.06.004

1037 Ones, D., Viswesvaran, C., \& Schmidt, F. (2008). No New Terrain: Reliability and Construct Validity of Job Performance Ratings. Industrial and Organizational Psychology, 1(2),

1040 Poropat, A. E. (2009). A meta-analysis of the five-factor model of personality and academic performance. Psychological Bulletin, 135(2), 322. https://doi.org/10.1037/a0014996

1042 Preacher, K. J., Zhang, G., Kim, C., \& Mels, G. (2013). Choosing the optimal number of factors 1043 in exploratory factor analysis: A model selection perspective. Multivariate Behavioral Research, 48(1), 28-56. https://doi.org/10.1080/00273171.2012.710386 Ziegler, M., Jones, A. B., \& Funder, D. C. (2014). The Situational Eight DIAMONDS: A taxonomy of major dimensions of situation characteristics. Journal of Personality and Social Psychology, 107(4), 677. https://doi.org/10.1037/a0037250 
1051 Reise, S. P., Moore, T. M., \& Haviland, M. G. (2010). Bifactor models and rotations: Exploring

1052

1053

1054

1055

1056

1057

1058

1059

1060

1061

1062

1063

1064

1065

1066

1067

1068

1069

1070

1071

1072 the extent to which multidimensional data yield univocal scale scores. Journal of Personality Assessment, 92, 544-559. http://dx.doi.org/10.1080/00223891.2010.496477

Rhoades, L., \& Eisenberger, R. (2002). Perceived organizational support: a review of the literature. Journal of Applied Psychology, 87(4), 698. https://doi.org/10.1037//0021$\underline{9010.87 .4 .698}$

Ribeiro, M. A., Teixeira, M. A. P., \& Ambiel, R. A. M. (2019). Decent work in Brazil: Context, conceptualization, and assessment. Journal of Vocational Behavior, 112, 229-240. https://doi.org/10.1016/j.jvb.2019.03.006

Rindskopf, D., \& Rose, T. (1988). Some theory and applications of confirmatory second-order factor analysis. Multivariate Behavioral Research, 23(1), 51-67. https://doi.org/10.1207/s15327906mbr2301_3

Roberts, B. W., Chernyshenko, O. S., Stark, S., \& Goldberg, L. R. (2005). The structure of conscientiousness: An empirical investigation based on seven major personality questionnaires. Personnel Psychology, 58(1), 103-139. https://doi.org/10.1111/j.1744$\underline{6570.2005 .00301 . x}$

Roberts, B. W., Lejuez, C., Krueger. R. F., Richards, J. M., Hill, P. L. (2014) What is Conscientiousness and how can it be assessed? Developmental Psychology 50(5), 1315 1330. https://doi.org/10.1037/a0031109

Rodriguez, A., Reise, S. P., \& Haviland, M. G. (2016). Evaluating bifactor models: Calculating and interpreting statistical indices. Psychological Methods, 21(2), 137. https://doi.org/10.1037/met0000045 
1073 Rozeboom, W. W. (1992). The glory of suboptimal factor rotation: Why local minima in analytic 1074 optimization of simple structure are more blessing than curse. Multivariate Behavioral Research, 27(4), 585-599. https://doi.org/10.1207/s15327906mbr2704 5

1076 Ruscio, J., \& Roche, B. (2012). Determining the number of factors to retain in an exploratory factor analysis using comparison data of known factorial structure. Psychological Assessment, 24(2), 282-292. https://doi.org/10.1037/a0025697

Schmid, J., \& Leiman, J. M. (1957). The development of hierarchical factor solutions. Psychometrika, 22(1), 53-61. https://doi.org/10.1007/bf02289209

Schmidt, F. L. (1971). The relative efficiency of regression and simple unit predictor weights in applied differential psychology. Educational and Psychological Measurement, 31(3), 699-714. https://doi.org/10.1177/001316447103100310

1084 Seltzer, B. K., Ones, D. S., \& Tatar, A. (2017). Using personality facets to understand the nature 1085 of personality-satisfaction relationships: Findings from meta-analytic bifactor latent modeling. Career Development International, 22(5), 477-506. https://doi.org/10.1108/cdi-08-2017-0141

1088 Smither, J. W., London, M., \& Reilly, R. R. (2005). Does performance improve following multisource feedback? A theoretical model, meta-analysis, and review of empirical findings. Personnel Psychology, 58(1), 33-66. https://doi.org/10.1111/j.1744-

1092 Spearman, C. (1904). "General intelligence," objectively determined and measured. The 1093 American Journal of Psychology, 15(2), 201-292. https://doi.org/10.2307/1412107

1094 Stanek, K. C., \& Ones, D. S. (2018). Taxonomies and compendia of cognitive ability and 1095 personality measures relevant to Industrial, Work, and Organizational Psychology. In The 
1096

1097

1098

1099

1100

1101

1102

1103

1104

1105

1106

1107

1108

1109

1110

1111

1112

1113

1114

1115

1116

SAGE Handbook of Industrial, Work and Organizational Psychology (Vol. 1). Sage London, United Kingdom. https://doi.org/10.4135/9781473914940.n14

Stanek, K. C., Ones, D. S., \& McGue, M. (2017). Counterproductive behaviors: Relations across life domains, etiology, and implications for applied practice. International Journal of Selection and Assessment 25, 111-124. https://doi.org/10.1111/ijsa.12164

Steiger, J. H. (1979). Factor indeterminacy in the 1930's and the 1970's some interesting parallels. Psychometrika, 44(2), 157-167. https://doi.org/10.1007/bf02293967

Steiger, J. H., \& Schönemann, P. H. (1978). A history of factor indeterminacy. In S. Shye (Ed.), Theory Construction and Data Analysis in the Behavioral Sciences, 136-178. San Francisco, CA: Jossey-Bass.

ten Berge, J. M., Krijnen, W. P., Wansbeek, T., \& Shapiro, A. (1999). Some new results on correlation-preserving factor scores prediction methods. Linear Algebra and its Applications, 289(1-3), 311-318. https://doi.org/10.1016/s0024-3795(97)10007-6

ten Berge, J. M. F. \& Sočan, G. (2004). The greatest lower bound to the reliability of a test and the hypothesis of unidimensionality. Psychometrika, 69(4), 613-625. https://doi.org/10.1007/BF02289858

Thomson, G. H. (1951). The factorial analysis of human ability. Houghton Mifflin.

Thurstone, L. L. (1934). The Vectors of Mind. Psychological Review, 41(1), 1-32. https://doi.org/10.1037/h0075959

Thurstone, L. L. (1947). Multiple-factor analysis; a development and expansion of the vectors of mind. University of Chicago Press. 
1117 Toker, Y., \& Ackerman, P. L. (2012). Utilizing occupational complexity levels in vocational

1118 interest assessments: Assessing interests for STEM areas. Journal of Vocational

1119 Behavior, 80(2), 524-544. https://doi.org/10.1016/j.jvb.2011.09.001

1120 Tucker, L. R. (1971). Relations of factor score estimates to their use. Psychometrika, 36(4), 427-

1121 436. https://doi.org/10.1007/bf02291367

1122 Viswesvaran, C., \& Ones, D. S. (2000). Perspectives on models of job performance.

1123 International Journal of Selection and Assessment, 8(4), 216-226.

1124 https://doi.org/10.1111/1468-2389.00151

1125 Viswesvaran, C., Ones, D. S., \& Schmidt, F. L. (1996). Comparative analysis of the reliability of

1126 job performance ratings. Journal of Applied Psychology, 81(5), 557-574.

1127 https://doi.org/10.1037/0021-9010.81.5.557

1128 Waller, N. G. (2018). Direct Schmid-Leiman transformations and rank-deficient loadings

1129 matrices. Psychometrika, 83(4), 858-870. https://doi.org/10.1007/s11336-017-9599-0

1130 Waller, N. G. (2019). Fungible: Psychometric functions from the Waller lab. R package version $1131 \quad 1.93$.

1132 Watson, D., Clark, L. A., \& Tellegen, A. (1988). Development and validation of brief measures

1133 of positive and negative affect: the PANAS scales. Journal of personality and social

1134 psychology, 54(6), 1063. https://doi.org/10.1037//0022-3514.54.6.1063

1135 Wherry, R. J. (1959). Hierarchical factor solutions without rotation. Psychometrika, 24(1), 45-

1136 51. https://doi.org/10.1007/bf02289762

1137 Wilmot, M. P., \& Ones, D. S. (2019). A century of research on conscientiousness at work.

1138 Proceedings of the National Academy of Sciences, 116(46), 23004-23010.

1139 https://doi.org/10.1073/pnas.1908430116 
1140 Wilson, E. B. (1928). On hierarchical correlation systems. Proceedings of the National Academy 1141 of Sciences of the United States of America, 14(3), 283.

$1142 \quad$ https://dx.doi.org/10.1073\%2Fpnas.14.3.283

1143 Wood, J. M., Tataryn, D. J., \& Gorsuch, R. L. (1996). Effects of under- and overextraction on 1144 principal axis factor analysis with varimax rotation. Psychological Methods, 1(4), 3541145 365. https://doi.org/10.1037//1082-989x.1.4.354

1146 Yang, R., Spirtes, P., Scheines, R., Reise, S. P., \& Mansolf, M. (2017). Finding pure submodels 1147 for improved differentiation of bifactor and second-order models. Structural Equation 1148 Modeling: A Multidisciplinary Journal, 24(3), 402-413. https://doi.org/10.1080/10705511.2016.1261351

1150 Yates, A. (1987). Multivariate exploratory data analysis: A perspective on exploratory factor 1151 analysis. Albany, NY: SUNY Press.

1152 Yung, Y.-F., Thissen, D., \& McLeod, L. D. (1999). On the relationship between the higher-order 1153 factor model and the hierarchical factor model. Psychometrika, 64(2), 113-128. 1154 https://doi.org/10.1007/bf02294531

1155 Zacher, H. (2014). Career adaptability predicts subjective career success above and beyond personality traits and core self-evaluations. Journal of Vocational Behavior, 84(1), 21-30.

1158 Zinbarg, R. E., Revelle, W., Yovel, I., \& Li, W. (2005). Cronbach's $\alpha$, Revelle's $\beta$, and 1159 McDonald's $\omega_{\mathrm{h}}$ : Their relations with each other and two alternative conceptualizations of 1160 reliability. Psychometrika, 70(1), 123-133. https://doi.org/10.1007/s11336-003-0974-7 


\section{Tables}

Table 1

Exploratory Bifactor Analysis Methods

\section{Analytic Strategy}

Model type Hybrid methods

Target rotation

Analytic bifactor rotation

\section{- Schmid- • Direct Schmid-Leiman}

\begin{tabular}{|c|c|c|c|}
\hline Hierarchical & $\begin{array}{l}\text { Leiman (1957) } \\
\text { • Wherry (1959) }\end{array}$ & (Waller, 2018) & $\begin{array}{c}\text { No Methods Currently } \\
\text { Available }\end{array}$ \\
\hline Non-hierarchical & $\begin{array}{l}\text { - Holzinger and } \\
\text { Swineford } \\
\text { (1937) }\end{array}$ & $\begin{array}{l}\text { - Direct Bifactor (Waller, } \\
\text { 2018) } \\
\text { - Schmid-Leiman target } \\
\text { rotation (Reise, Moore, \& } \\
\text { Haviland, 2010) } \\
\text { - Iterated Schmid-Leiman } \\
\text { target rotation (Abad et al., } \\
\text { 2017) }\end{array}$ & $\begin{array}{l}\text { - Bifactor Quartimin } \\
\text { (Jennrich \& Bentler, } \\
\text { 2011, 2013) } \\
\text { - Bifactor Geomin } \\
\text { (Jennrich \& Bentler, } \\
\text { 2012) }\end{array}$ \\
\hline
\end{tabular}

Note: Bolded methods have been found to accurately recover the loadings matrix of bifactor measurement models (Giordano \& Waller, 2020). 
Table 2

Schmid-Leiman Bifactor Solution of the Multisource Performance Ratings

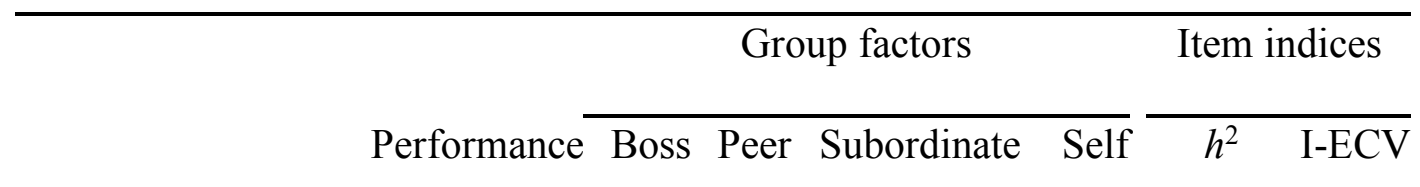

\section{Boss Ratings}

Technical

$\begin{array}{lll}.48 \quad .86 & -.01\end{array}$

$-.01 \quad .00 \quad .97$

.24

Interpersonal

$.48 \quad .78 \quad .01$

$\begin{array}{llll}.02 & -.01 & .84\end{array}$

.27

Leadership

$.45 \quad .76 \quad .00$

$\begin{array}{lll}.00 & .02 \quad .77\end{array}$

.26

Peer Ratings

Technical

$.58 \quad .00 \quad .78$

$\begin{array}{lll}-.02 & .01 & .95\end{array}$

.36

Interpersonal

$.56 \quad .00 \quad .72$

$\begin{array}{lll}.01 & -.02 & .83\end{array}$

.38

Leadership

$\begin{array}{lll}.53 & .01 \quad .65\end{array}$

$.03 \quad .02 \quad .70$

\section{Subordinate Ratings}

Technical

Interpersonal

Leadership

Self-Ratings

Technical

Interpersonal

Leadership $\begin{array}{lll}.59 & -.01 & -.02\end{array}$

$\begin{array}{lll}.56 & .01 & .02\end{array}$

$\begin{array}{lll}.51 & .01 \quad .01\end{array}$

$\begin{array}{lll}.63 & .01 & 67\end{array}$

$.77 \quad .01 \quad .94$

.36

.40

Note: $h^{2}=$ indicator communality; I-ECV = item (indicator) explained common variance. 


\section{Table 3}

Direct Schmid-Leiman Bifactor Solution of the Conscientiousness Subscales

\begin{tabular}{|c|c|c|c|c|c|}
\hline & \multirow[b]{2}{*}{ Conscientiousness } & \multicolumn{2}{|c|}{ Group Factors } & \multicolumn{2}{|c|}{ Item indices } \\
\hline & & PWO & Conformity & $h^{2}$ & I-ECV \\
\hline Diligence & .58 & .70 & .02 & .82 & .41 \\
\hline Achievement & .55 & .67 & .01 & .74 & .40 \\
\hline Persistence & .56 & .64 & .05 & .73 & .42 \\
\hline Industriousness & .37 & .44 & .03 & .33 & .42 \\
\hline Virtue & .51 & .43 & .25 & .51 & .51 \\
\hline Deliberateness & .42 & .41 & .14 & .36 & .49 \\
\hline Cautiousness & .41 & .31 & .24 & .32 & .52 \\
\hline Orderliness & .37 & .29 & .22 & .27 & .52 \\
\hline Dutifulness & .55 & .03 & .83 & .99 & .31 \\
\hline Traditionalism & .29 & .04 & .40 & .25 & .34 \\
\hline Responsibility & .41 & .22 & .36 & .35 & .49 \\
\hline
\end{tabular}

Note: $\mathrm{PWO}=$ Prudent work orientation; $h^{2}=$ indicator communality; I-ECV $=$ item (indicator) explained common variance. 


\section{Table 4}

Intercorrelations Between Factor Score Estimates in the Conscientiousness Bifactor Model

$$
\text { Prudent work }
$$

Conscientiousness orientation Conformity

$\begin{array}{lccc}\text { Conscientiousness } & (.96) & .63 & .52 \\ \text { Prudent work orientation } & .97 & (.85) & -.34 \\ \text { Conformity } & .67 & .51 & (.74)\end{array}$

Note: Values in the lower triangle represent the correlations between unit-weighted factor score estimates; values in the upper triangle represent the correlations between Thurstone's regression-based factor score estimates. Values in the matrix diagonal represent the correlation of scores on the same factor by different factor scoring methods. 
Figure 1

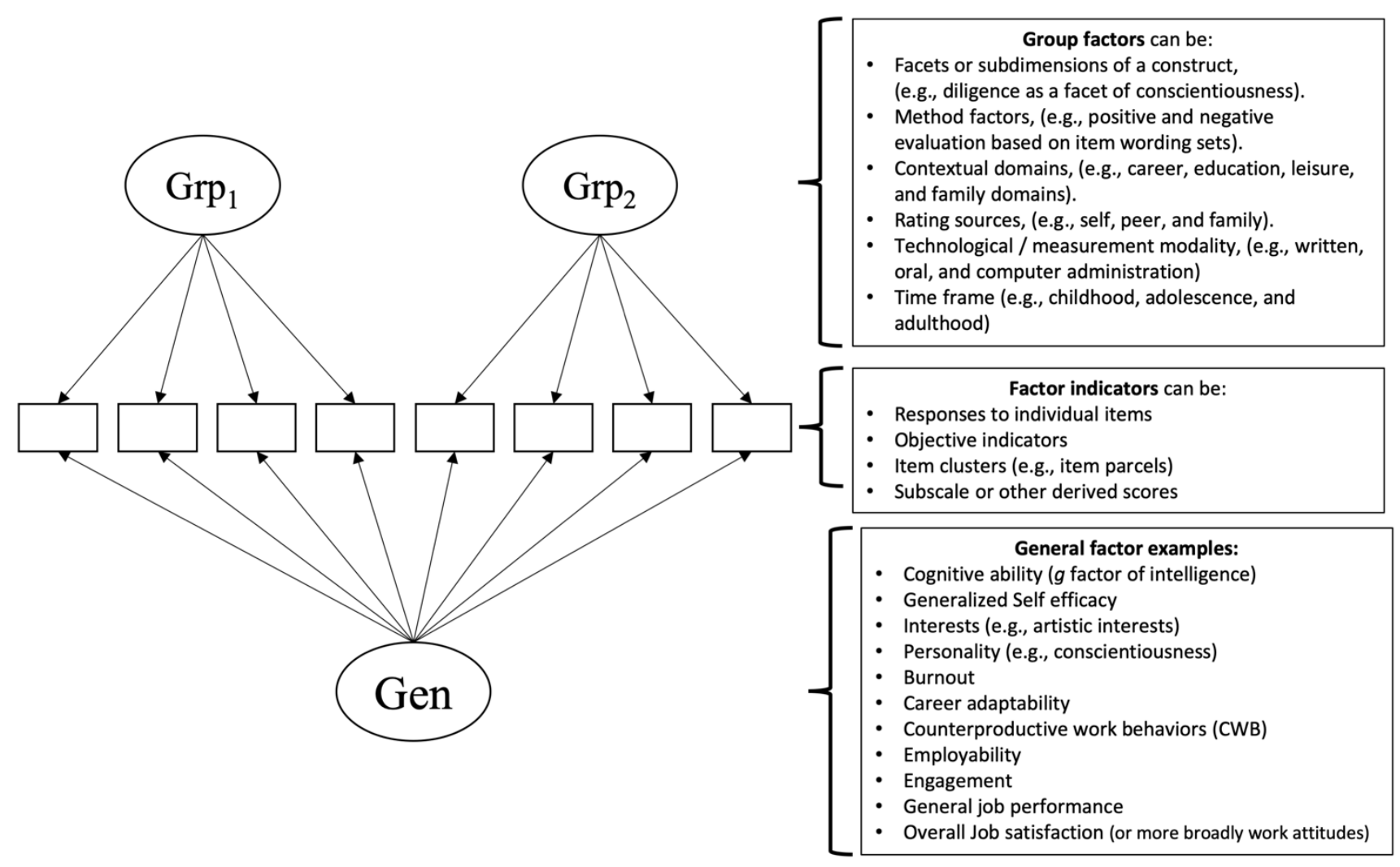

caption: The diagram on the left-hand side of the figure depicts a (hierarchical or non-hierarchical) bifactor model with a general factor (i.e., the circle labeled 'Gen') and two group factors (i.e., the circles labeled 'Grp 1 ' and 'Grp 2 '). Boxes represent the factor indicators. On the right-hand side of the figure, text boxes contain (non-exhaustive) example applications for modeling the group factors, types factor indicators, and example constructs in which to model a general factor. 
Figure 2

Comparing Oblique Rotations in the SL procedure

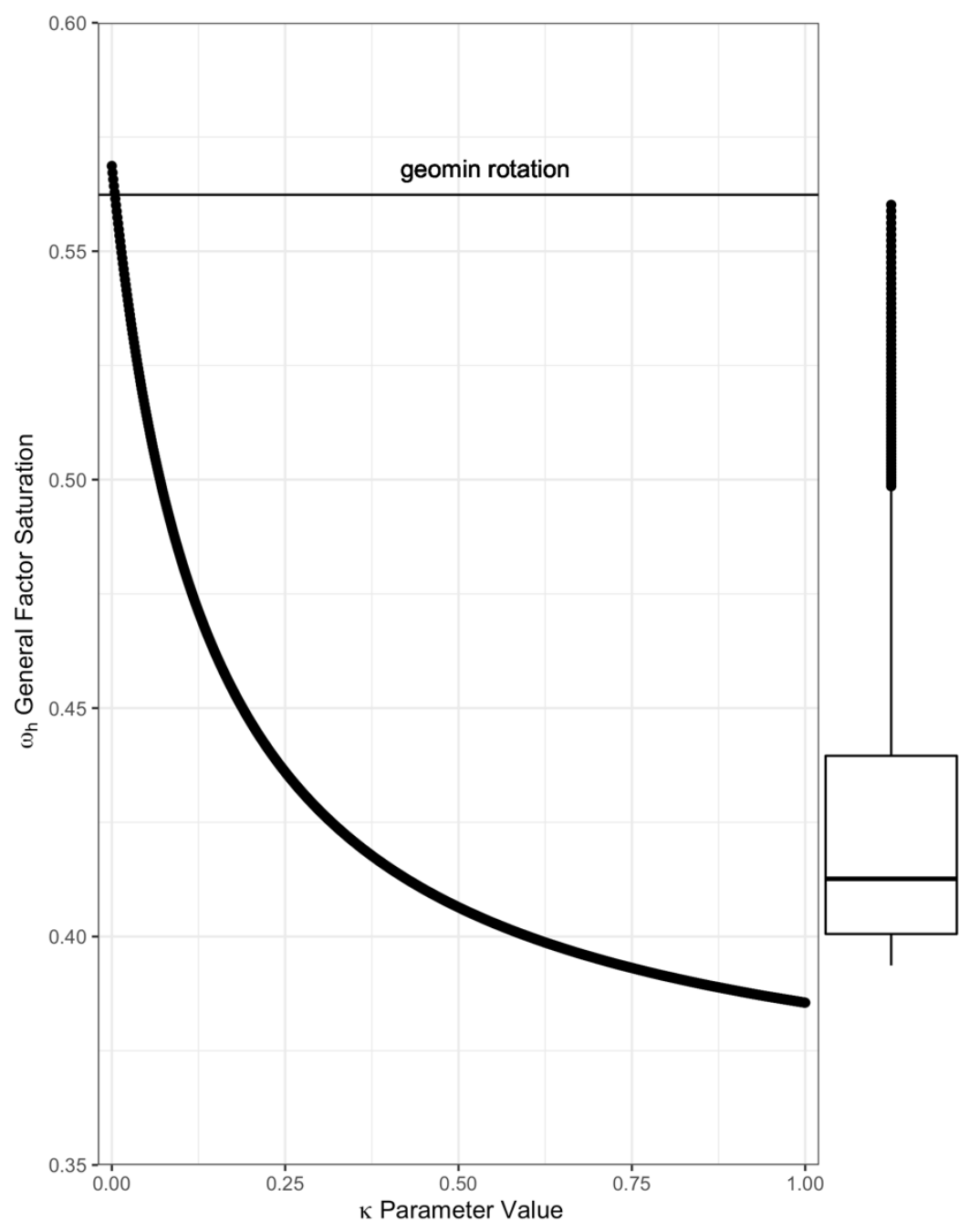

Caption: Using data from Hoffman et al. (2010), general factor saturation $\left(\omega_{h}\right)$ for each of the 1,001 rotations is plotted against the rotation tuning parameter ( $\kappa$; Crawford \& Ferguson, 1970). The solid horizontal line depicts the general factor saturation obtained from a geomin rotation $\left(\omega_{h}=.56\right)$ as a point of reference. General factor saturation ranges from $38.55 \%$ to $56.87 \%$ of the total sum score variance. 